\title{
Dual drug-loaded nanoparticles on self-integrated scaffold for controlled delivery
}

This article was published in the following Dove Press journal:

International Journal of Nanomedicine

13 July 2012

Number of times this article has been viewed

\author{
Devasier Bennet' \\ Mohana Marimuthu' \\ Sanghyo Kim' \\ Jeongho $\mathrm{An}^{2}$ \\ 'Department of Bionanotechnology, \\ Gachon University, Gyeonggi, \\ Republic of Korea; ${ }^{2}$ Department of \\ Polymer Science and Engineering, \\ SunKyunKwan University, Gyeonggi, \\ Republic of Korea
}

Correspondence: Sanghyo Kim

Department of Bionanotechnology,

Gachon University, San 65,

Bokjeong-Dong, Sujeong-Gu, Seongnam-Si, Gyeonggi-Do 46I-70I, Republic of Korea

Tel +8I3 I750 8554

Fax +8I3 I750 88I9

Email samkim@kyungwon.ac.kr

Jeongho An

Department of Polymer Science and

Engineering, SunKyunKwan University,

Suwon, Gyeonggi-Do 440-I46, Republic of Korea

Tel +823 I290 7284

$\mathrm{Fax}+823 \quad I 2928790$

Email jhahn I us@skku.edu
Abstract: Antioxidant (quercetin) and hypoglycemic (voglibose) drug-loaded poly-D,L-lactideco-glycolide nanoparticles were successfully synthesized using the solvent evaporation method. The dual drug-loaded nanoparticles were incorporated into a scaffold film using a solvent casting method, creating a controlled transdermal drug-delivery system. Key features of the film formulation were achieved utilizing several ratios of excipients, including polyvinyl alcohol, polyethylene glycol, hyaluronic acid, xylitol, and alginate. The scaffold film showed superior encapsulation capability and swelling properties, with various potential applications, eg, the treatment of diabetes-associated complications. Structural and light scattering characterization confirmed a spherical shape and a mean particle size distribution of $41.3 \mathrm{~nm}$ for nanoparticles in the scaffold film. Spectroscopy revealed a stable polymer structure before and after encapsulation. The thermoresponsive swelling properties of the film were evaluated according to temperature and $\mathrm{pH}$. Scaffold films incorporating dual drug-loaded nanoparticles showed remarkably high thermoresponsivity, cell compatibility, and ex vivo drug-release behavior. In addition, the hybrid film formulation showed enhanced cell adhesion and proliferation. These dual drugloaded nanoparticles incorporated into a scaffold film may be promising for development into a transdermal drug-delivery system.

Keywords: quercetin, voglibose, biocompatible materials, encapsulation, transdermal

\section{Introduction}

Oxidative stress plays a major role in the pathogenesis of diabetes-associated cardiovascular disease. ${ }^{1-3}$ Regulation of antioxidants is the main difficulty in the clinical treatment of diabetes and its complications. ${ }^{4}$ It is clear that strategies to block the formation of reactive radicals by providing antioxidants would enable a targeted approach to treating the complications of diabetes. ${ }^{5,6}$ Despite the introduction of new therapies, most of the research so far has been focused on conventional treatment alternatives. Combination therapy using different drugs is a promising strategy to suppress diabetes-associated cardiovascular disease and may induce synergism. Further, dual therapy may prevent secondary damage to cells and recurrence of disease. ${ }^{3}$ Therapeutics research should be focused on improving the treatment of diabetic patients with oxidative stress damage, immunodeficiency disease, or cardiovascular disease. ${ }^{7,8}$ The mechanisms of acceleration of oxidative stress should be evaluated for development of multimodal treatment options in diabetic populations. ${ }^{4,5,9}$ The oral route of drug administration has several disadvantages, including first-pass metabolism and low intestinal bioavailability, as well as an inability to control drug delivery or regulate the rate of absorption. ${ }^{10,11}$ These problems could be overcome by development of a novel combined therapy to improve the bioavailability of 
antioxidant and hypoglycemic drugs via a transdermal delivery system. ${ }^{12,13}$ A transdermal delivery system is an appropriate route for administration of free radical scavengers because skin is an important target candidate for oxidative stress and the combined therapy can be good as an antidote to reduce secondary damage. ${ }^{5,6}$ Most importantly, a transdermal drugdelivery system can prevent first-pass metabolism, enabling high bioavailability of drugs and enhance patient compliance, with fewer doses needed and minimal toxicity.

A more complete coverage approach designed to inhibit production of reactive oxygen species could be beneficial. The choice and dose of an antioxidant using an alphaglucosidase inhibitor could play a major role in combination therapy and could lead to reduction of oxidative stress and hyperglycemia. ${ }^{3,14-16}$ Voglibose is a third-generation alpha-glucosidase inhibitor with hypoglycemic activity, and quercetin is a well established natural flavone with antioxidant properties. Quercetin has high potency in several kinds of combined drug delivery. ${ }^{17}$ Therefore, nanoparticles encapsulating a combination of drugs hold promise for multifunctional application. ${ }^{13,18-22}$ Many researchers have demonstrated that simultaneous administration of antioxidants and hypoglycemic drugs can result in better treatment efficacy. ${ }^{3}$ Furthermore, poly-D,L-lactide-co-glycolide (PLGA)based nanoparticles may enhance combined drug delivery. However, impermeability of human skin is a major problem that has to be overcome for transdermal drug-delivery system applications. ${ }^{23}$ Oleic acid has been extensively studied for its chemical permeation enhanced activity in increasing drug diffusion across the skin. Utilization of chemical permeation enhancers in preparation of nanoparticles can overcome the barrier function of skin. ${ }^{24-26}$

Different types of film dressing materials are available, including chitin, chitosan, Tegaderm ${ }^{\mathrm{TM}}$ (3M Health Care, Borken, Germany), hydrocolloid, biofilm, and alginate. ${ }^{27,28}$ Alginate film is the most widely recommended dressing in the diabetic population, having a good safety profile and being suitable for application on wounds. An optimal skin film candidate for a transdermal delivery system should not only hold the active drug but have a good cosmetic appearance and be able to hold functional encapsulated polymer nanoparticles for controlled drug release. Polymers have enhanced water solubility under increased temperature conditions, but have decreased water solubility in the presence of a cross-linking agent. It has been demonstrated that polymer films containing amphiphilic (hydrophilic and hydrophobic) groups improve controlled transdermal drug release in response to temperature changes.
In the present study, an attempt was made to achieve swelling-induced dual drug release from a nanoparticleloaded scaffold film. A solvent evaporation method was used to encapsulate quercetin and voglibose as the functional drugs into PLGA nanoparticles, which were later incorporated into the scaffold film. Using a solvent casting method, the self-assembled biological scaffold film was prepared using various ratios of excipients, ie, sodium alginate, polyvinyl alcohol, hyaluronic acid, xylitol, and polyethylene glycol-600, in order to attain thermosensitive properties. ${ }^{29}$ Furthermore, the excipients used showed key pharmaceutical properties, including biocompatibility, safety, ${ }^{30}$ anti-infective properties, controlled release, ${ }^{31,32}$ flexibility, emollient, adhesion, spreadability, and retention ability for film formulation. ${ }^{33-35}$ The physicochemical properties, drug release, and cell compatibility characteristics of the scaffold film were studied. It was expected that this film has the potential to enable controlled drug delivery across the skin for prolonged time periods.

\section{Materials and methods Materials}

Quercetin, hyaluronic acid, PLGA (50:50 molecular weight $15 \mathrm{kDa})$, voglibose, xylitol, sodium alginate, Tween-20, 3-(4,5-dimethylthiazolyl)-2,5-diphenyltetrazolium bromide, and oleic acid were purchased from Sigma-Aldrich Chemical Company Ltd (St Louis, MO). Polyvinyl alcohol-500 was purchased from Junsei Chemical Company Ltd (Tokyo, Japan). Polyethylene glycol-600 was purchased from Daejung Chemicals and Metals Company Ltd (Gyeonggi-do, South Korea). Cadaver skin (from the rear thigh of a 40-yearold male) was purchased from Hans Biomed Corporation (Seoul, South Korea). Dulbecco's modified Eagle's medium, fetal bovine serum, trypsin/ethylenediaminetetraacetic acid, and penicillin-streptomycin were purchased from Gibco Laboratories (Grand Island, NY). Trypan blue dye was purchased from Alfa Aesar Company (Ward Hill, MA). All other chemicals used were of pharmaceutical grade. Milli-Q water (18.2 M $\Omega$; Millipore Corporation, Billerica, MA) was used throughout the experiments.

\section{Preparation of PLGA nanoparticles containing quercetin and voglibose}

Drug-loaded PLGA nanoparticles were prepared by the solvent evaporation method according to previous reports, ${ }^{36-39}$ with slight modification. The organic phase of quercetin $0.05 \mathrm{~g}$, voglibose $0.01 \mathrm{~g}$, and PLGA $0.25 \mathrm{~g}$ solubilized in $3 \mathrm{~mL}$ of acetone was added dropwise into $30 \mathrm{~mL}$ of the aqueous phase containing $0.194 \mathrm{~g}$ of Tween-20 
(as emulsifier) under magnetic stirring at $2500 \mathrm{rpm}$ for one hour to generate a PLGA-drug nanoemulsion. Subsequently, oleic acid (at ratios of 4\%, 4.5\%, 5\%, 5.5\%, and 6\% w/v) was added to the crude emulsion, which was then sonicated for 5 minutes with a probe sonicator (VCX750; Sonics and Materials Inc, Danbury, CT) at an optimal amplitude of $40 \%$ and a frequency of $20 \mathrm{kHz}$. Sonication is a crucial step in preparation of a nanoemulsion, and can increase the temperature to that needed to inactivate antioxidants. Therefore, we utilized an on/off cycle to maintain a low temperature. The solution gradually changed from clear light yellow to milk-white yellow. Oleic acid acted as both a chemical permeation enhancer and as a surfactant to obtain a homogeneous nanoemulsion. ${ }^{25,40}$ The nanoemulsion was diluted by adding water to make a final formulation of $70 \mathrm{~mL}$. The acetone was removed, and the aqueous phase was concentrated by rotary evaporation at $40^{\circ} \mathrm{C}$ under reduced pressure. The concentrated nanoemulsion contained nanoparticles of the smallest size. These nanoparticles were centrifuged, washed, freeze-dried, and stored at room temperature for further experiments.

\section{Characterization of nanoparticles}

The effects of various process parameters and polymer characteristics on the formation of nanoparticles were evaluated. The morphology and size distribution were studied using field emission scanning electron microscopy (JEOL-ISM-7500F; JEOL, Tokyo, Japan), and photon correlation spectroscopy, respectively. The particle size distribution of the PLGA nanoparticles was determined using a Malvern Zetasizer Nano ZS (Malvern Instruments, Worcestershire, UK), with a laser at $633 \mathrm{~nm}, 25^{\circ} \mathrm{C}$, and a scattering angle of $90^{\circ}$. The particle dispersions were diluted 1:10 with the aqueous phase of the formulations to get suitable kilocounts per second. The polydispersity index measures the size distribution of the population. All batches of nanoparticles were produced in triplicate at least.

\section{Drug entrapment efficiency}

The freeze-dried nanoparticles recovered were dissolved in $50 \mathrm{~mL}$ of dimethyl sulfoxide and then subjected to ultracentrifugation for one hour at 20,000 rpm. The amounts of drugs present in the supernatant were analyzed using an ultraviolet-visible spectrophotometer (Optizen 3220; Mecasys, Daejeon, Korea) at the highest spectrum wavelengths of $282 \mathrm{~nm}$ and $374 \mathrm{~nm}$. A standard calibration curve was constructed, using different concentrations of voglibose and quercetin $(0.01-12.5 \mu \mathrm{g})$ versus maximum absorbance. For better estimation prior to measurement, a voglibose solution was derivatized using taurine and sodium periodate in water and methanol. The maximum absorbance of the supernatants of drug-loaded nanoparticles was used for quantification of voglibose and quercetin at wavelengths of $282 \mathrm{~nm}$ and $374 \mathrm{~nm}$, respectively. ${ }^{41,42}$ The percentages of nanoparticle recovery, drug content, and drug entrapment were determined using equations 1,2 , and 3, respectively. The experiments were performed in triplicate and reported to establish reproducibility of synthesis.

Nanoparticle recovery (\%)

$$
=\frac{\text { Mass of nanoparticle recovered }}{\text { Mass of polymeric particles, drug and }} \times 100
$$

$$
\begin{aligned}
& \text { Drug content }(\%, w / w) \\
& =\frac{\text { Mass of drug in nanoparticle }}{\text { Mass of nanoparticle recovered }} \times 100
\end{aligned}
$$

Drug entrapment (\%)

$$
=\frac{\text { Mass of drug in nanoparticle }}{\text { Mass of drug used in nanoparticle formulation }} \times 100
$$

\section{Preparation of hybrid film-forming gels}

The films were prepared from aqueous solutions of filmforming gels which were synthesized according to a method reported previously, ${ }^{43}$ with slight modification. Different ratios of polymer composition were added to make a jelly solution, and are described in Table 1. Polyvinyl alcohol was dissolved in deionized water at $90^{\circ} \mathrm{C}$ to obtain a clear solution followed by addition of polyethylene glycol-600 to obtain a thermoresponsive gel. Sodium alginate was blended with hyaluronic acid to yield the swelling gel and xylitol was added into the solution for anti-infective capability. ${ }^{31}$ Finally, polyvinyl alcohol-polyethylene glycol-600 solution was added to the above solution of sodium alginate-hyaluronic acid-xylitol under constant stirring at room temperature for one hour.

Table I Composition of mixtures used for film preparation (w/w\%)

\begin{tabular}{llllll}
\hline Ingredients & S1 & S2 & S3 & S4 & Blank \\
\hline Drug-loaded nanoparticles & 2 & 2 & 2 & 2 & - \\
Sodium alginate & 25 & 20 & 15 & 10 & 20 \\
Polyvinyl alcohol & 10 & 15 & 20 & 25 & 15 \\
Hyaluronic acid & 3 & 3 & 3 & 3 & 3 \\
Xylitol & 5 & 5 & 5 & 5 & 5 \\
Polyethylene glycol-600 & 10 & 10 & 10 & 10 & 10 \\
Water & 45 & 45 & 45 & 45 & 47 \\
\hline
\end{tabular}


Polymers were used for the gel preparation to increase water solubility as the temperature increased and hence polymers had to be modified with a cross-linking agent to control solubility in water. Therefore, the gel was dissolved in $3 \mathrm{~mL}$ of $50 \mathrm{mM}$ 1-ethyl-3-(3-dimethylaminopropyl) carbodiimide (EDC) solution and stirred for 12 hours at room temperature to obtain a clear homogeneous solution. The gel was also prepared without a cross-linking agent as a blank. The resulting gel was stored in glass vials and sealed tightly with a siliconized rubber plug and an aluminum cap until use.

\section{Fabrication of nanoparticle-loaded scaffold film}

The scaffold film was prepared using a solvent casting method. ${ }^{44}$ The freeze-dried nanoparticles were added slowly into the gel and stirred for 4 hours at room temperature. The gel-nanoparticle solution was then poured into a clean dry Petri dish and kept under vacuum (in a dust-free atmosphere) for removal of air bubbles, followed by drying overnight. The resulting gel was cast as a dried backing layer. The dried nanoparticle layer incorporated into the scaffold film was carefully peeled off and stored at room temperature in a desiccator. A scaffold film without crosslinking agents or nanoparticles was also prepared and used as a blank. The resulting nanoparticle-loaded scaffold films are shown as $\mathrm{S} 1$, S2, S3, and S4, based on film composition, in Table 1.

\section{Morphology and topography}

The morphology and topography of the nanoparticle-loaded scaffold films were analyzed before and after application, using both optical and field emission scanning electron microscopy. Prior to examination by field emission scanning electron microscopy, the specimens were mounted on doublesided adhesive carbon tapes in a metal stub, sputter-coated for 80 seconds with platinum under vacuum to avoid accumulation of charge, and examined at $15 \mathrm{kV}$. The surface morphology and roughness of the scaffold films were observed before and after application using bioatomic force microscopy (Nanowizard II; JPK Instruments, Berlin, Germany) in intermittent air mode, with the root mean square value obtained from the JPK software. The dry films were scanned using a $\mathrm{Si}_{3}$ $\mathrm{N}_{4}$ cantilever with the scan speed of $0.5-1.5 \mathrm{~Hz}$. A large scan area $(1 \times 1 \mu \mathrm{m})$ was utilized to investigate the homogeneity and regularity of the polymer film surface.

\section{Structural characterization of scaffold film}

Fourier-transform infrared spectroscopy (FT-IR) was used to detect fingerprint changes in the functional groups and the chemical stability of the samples (ie, drugs, polymer, drug-loaded nanoparticles, and film). The final scaffold films were recorded at room temperature using a Tensor 27 instrument (Bruker Optics, Woodlands, TX) in the region of 500-4000 $\mathrm{cm}^{-1}$. Prior to FT-IR examination, the specimens were placed in a sample holder on the path to obtain their spectra. Differential scanning calorimetry and thermogravimetric analysis (SDT Q600; TA Instruments, Seoul, Korea) were used to study the thermal stability of the films. The samples were first heated at a rate of $5^{\circ} \mathrm{C}$ per minute in a temperature range of $20^{\circ} \mathrm{C}-110^{\circ} \mathrm{C}$ under a nitrogen atmosphere. An X-ray diffractometer (Model D/Max-2200; Rigaku, Woodlands, TX) with a positionsensitive detector was used. $\mathrm{CuK}$ alpha radiation at a wavelength $1.5406 \AA$ was used to characterize the crystalline properties of the films from $10^{\circ}$ to $90^{\circ}$ at a scan interval of 0.02 seconds.

\section{Swelling properties}

Environmental effects on the swelling properties of the films were investigated using a modified weighing method. ${ }^{44,45}$ Swelling behavior was determined by varying the ratios of the polymers, temperature, and $\mathrm{pH}$, and the results were compared with the noncross-linked blank scaffold films. The films were immersed in solutions to swell at different temperatures of $27^{\circ} \mathrm{C}, 37^{\circ} \mathrm{C}$, and $47^{\circ} \mathrm{C}$, and at different $\mathrm{pH}$ levels of 5.5, 6.5, and 7.5. Monobasic, dibasic, and tribasic sodium phosphate solutions were used to adjust the $\mathrm{pH}$. At different time intervals, samples were taken from the solutions, and weighed after excess solution on the surfaces had been blotted (in a 75\% relative humidity chamber for 30 minutes). The films were dried in a vacuum desiccator for at least 24 hours and weighed. The swelling ratio $\left(Q_{s}\right)$ of the films was calculated as:

$$
\mathrm{Q}_{\mathrm{s}}=\left(\mathrm{W}_{\mathrm{s}}-\mathrm{W}_{\mathrm{d}}\right) / \mathrm{W}_{\mathrm{d}}
$$

where $\mathrm{W}_{\mathrm{d}}$ is the weight of the dry film and $\mathrm{W}_{\mathrm{s}}$ is the weight of the swollen film at certain time intervals.

\section{In vitro biocompatibility}

The biocompatibility of the films was investigated by MTT assay ${ }^{46}$ using human fibroblasts (FY11 cells). ${ }^{47}$ The cell culture was carried out using Dulbecco's modified Eagle's medium containing $10 \%$ fetal bovine serum, penicillin $10,000 \mathrm{U} / \mathrm{mL}$, and streptomycin $10 \mathrm{mg} / \mathrm{mL}$. Ultravioletsterilized blank and nanoparticle-loaded films $(10 \mathrm{~mm}$ in diameter) were placed in a 6-well plate and the cells were 
seeded at $6 \times 10^{5}$ per well. One hour prior to cell seeding, $1 \mathrm{~mL}$ of culture medium was added, and then incubated in normal growth conditions to allow growth for 3 days at $37^{\circ} \mathrm{C}$ in $5 \% \mathrm{CO}_{2}$ and $95 \%$ humidity. After 3 days of culture, cell viability was quantified using the MTT assay reagent at $5 \mathrm{mg} / \mathrm{mL}$. Cells cultured without films were considered as controls and the same procedure was followed for analysis of cell viability. Viability of the FY11 cells in the presence of the films was also observed by fluorescent microscopy and DAPI nuclear staining. After treating the cells with the films for 3 days, $0.1 \mathrm{mM}$ DAPI was added to each well and incubated for 15 minutes, and then viewed under a phase contrast fluorescent microscope to visualize the metabolically active cells.

\section{Ex vivo drug release study}

Dual drug-loaded nanoparticles incorporated into scaffold films and blank films $10 \mathrm{~mm}$ in diameter were used for the skin permeation study. ${ }^{48}$ This was performed using Franz diffusion apparatus with a $3 \mathrm{~cm}^{2}$ surface area. The receiving chamber was filled with phosphate-buffered solution and the study was performed under physiological conditions (medium containing 1\% Tween-20 which can increase solubility of drugs and sink conditions, and maintained with constant agitation and temperature $\left(37^{\circ} \mathrm{C} \pm 1^{\circ} \mathrm{C}\right)$ using a flow loop consisting of a water bath reservoir). Human cadaver skin was placed between the donor and receiver compartments, with the stratum corneum facing the donor compartment to make contact with the releasing surface of the system. The film was placed inside the donor chamber of the Franz diffusion apparatus, and $1.0 \mathrm{~mL}$ samples were then withdrawn from the receiver compartment $(5 \mathrm{~mL})$ at different time intervals and replaced with an equal amount of fresh medium. The samples collected were added to $5 \mathrm{~mL}$ of dimethyl sulfoxide solution, mixed together for one hour, and finally subjected to ultracentrifugation at 20,000 rpm for 30 minutes. The supernatant was collected and analyzed by ultraviolet-visible spectroscopy at an optical wavelength of $282 \mathrm{~nm}$ and $374 \mathrm{~nm}$ for voglibose and quercetin, respectively. The cumulative amount of drug permeation was measured using maximum absorbance at the particular wavelength of each drug. Cumulative amounts of drugs contained in the various formulations permeating the skin per unit surface area were plotted as a function of time.

\section{Statistical analysis}

All statistical calculations were performed using GraphPad software (La Jolla, CA). One-way analysis of variance was performed with the $95 \%$ confidence interval, followed by Tukey's multiple comparison tests, and the results are presented as the mean \pm standard deviation. $P<0.05$ was considered to indicate a statistically significant difference between the treatment groups. The error bars show the standard deviation ( $\mathrm{n}=3$ for all cases).

\section{Results and discussion Nanoparticle fabrication and morphological characterization}

The basic composition of a system containing a constant concentration of anionic surfactants and different oleic acid ratios was used to evaluate surface permeation enhanced formation of nanoparticles. Optimization and development of stable, uniform, and smooth nanoparticles could be achieved by modifying their composition using different concentrations of oleic acid (4, 4.5, 5, 5.5, and $6 \mathrm{wt} \%)$. Figure 1 shows images of the nanoparticle dispersions, indicating that the nanoparticles tended to aggregate when the concentration of oleic acid was increased from $5 \mathrm{wt} \%$ to $6 \mathrm{wt} \%$ (ie, an excess of oleic acid floated on the upper meniscus and bigger-sized aggregated particles settled down in a vertical bottle), whereas a low concentration of oleic acid (4 $\mathrm{wt} \%)$ produced undesirably smaller nanoparticles which floated on the surface of the solution. The concentrations of aqueous Tween-20 and organic phase polymer, as well as the defined synthesis parameters, were fixed, as described in our previous report. ${ }^{49}$ The diameter of the resulting nanoparticles decreased from $228.6(6 \mathrm{wt} \%$ oleic acid) to 38.5 (4 wt $\%$ oleic acid) nm upon modifying the oleic acid concentration (see supplementary information, Figure $\mathrm{S} 1$ ). The results show that a $4.5 \mathrm{wt} \%$ concentration

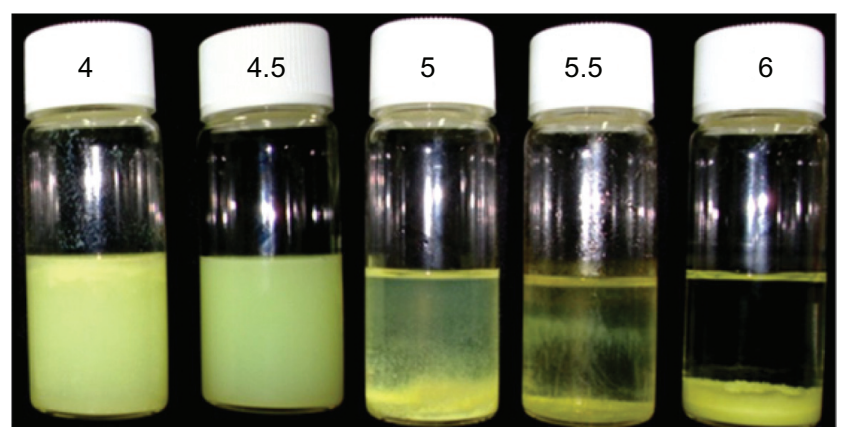

Figure I Stability of drug-loaded nanoparticles with different oleic acid concentrations $(4,4.5,5,5.5$, and 6 wt\%).

Notes: Suspension containing 4 wt\% oleic acid produced inappropriately small sized nanoparticles which floated on the surface of the solution. The suspension containing 4.5 wt\% oleic acid shows a uniform particle distribution without aggregation, whereas the nanoparticles tend to aggregate upon increasing the concentration of oleic acid from 5 wt $\%$ to 6 wt $\%$. 
of oleic acid forms homogeneously dispersed smooth nanoparticles without any aggregation. For physicochemical characterization of all the formulations used in our experiments, the purified nanoparticle dispersions were diluted 1:10 with the aqueous phase of the formulations to obtain suitable kilocounts per second. The sizes of the oleic acid nanoparticles in five different formulations with and without the chemical permeation enhancer are shown in Table S1 in the supplementary information. All formulations in the study had a uniform homogeneous size distribution with a polydispersity index of $0.34-0.11$ and a zeta potential between -46 and -56 . The zeta potential of $4.5 \mathrm{wt} \%$ oleic acid would promote high stability with a constant Tween-20 surfactant, and prevent aggregation of the nanoparticle system. The polydispersity index decreased with decreasing concentrations of oleic acid, indicating formation of monodispersed particles with a uniform size distribution. The increased ratio of oleic acid had an increased polydispersity index value, indicating that heterogeneity was gradually increasing in the nanoparticles. In addition, all ratios of oleic acid-containing nanoparticles were more uniform compared with those formed without oleic acid, hence the smaller difference in the polydispersity index value. Using oleic acid resulted in more stable particles because of incorporation of a hydrophobic core able to interact with the hydrophobic tail of the drug present in the particles with surfactant. This formulation showed excellent storage stability. Nanoparticles containing $4.5 \mathrm{wt} \%$ oleic acid showed no aggregation or separation, and had a uniform size distribution. Hence this preparation was selected for further study. Figure 2 shows the particle size distribution and field emission scanning electron microscopy images of nanoparticles with a diameter of $41.3 \mathrm{~nm}$.
Nanoparticle size depends mostly on the surfactant ratio (hydrophilic Tween-20 and lipophilic oleic acid).

\section{Effects of oleic acid ratio on nanoparticles}

The concentration of oleic acid alters the size and surface charge of the nanoparticles. The initial percentage of surfactant in the system increases upon addition of oleic acid, disturbing the formation of nanoparticles. The physicochemical characterization of drug-loaded nanoparticle preparations containing different ratios of oleic acid are shown in Table 1 in the supplementary information. Systems with more than $5 \mathrm{wt} \%$ oleic acid formed flocculations and, below $4.5 \mathrm{wt} \%$ oleic acid, the nanoparticles floated on the surface due to the variation in surfactant content. The appropriate nanoparticle formation is directly dependent on the concentration of oleic acid and surfactant. An increased oleic acid concentration enhances attachment of $\mathrm{COOH}$ groups onto the nanoparticle surface. Hence the surface charge of the nanoparticles formed is strongly negative as a result of deprotonation of the-OH group on oleic acid during formation. The polydispersity index results were similar for all formulations in the study, all being less than 0.339 with a high negative zeta potential. The mean polydispersity index for the $4.5 \mathrm{wt} \%$ oleic acid formulation was $0.118 \pm$ 0.013 with a mean negative zeta potential of $-47.3 \pm 2.4$. Of all the study formulations, the $4.5 \mathrm{wt} \%$ oleic acid formulation showed the best storage stability, with the particle size changing by only a few nanometers during 3 months of storage (data not shown), and other nanoparticles showing less stability and particle defects after this time. Table 1 shows the percentage drug content, entrapment, and recovery for the $4.5 \mathrm{wt} \%$ oleic acid formulation. The purified drug-loaded
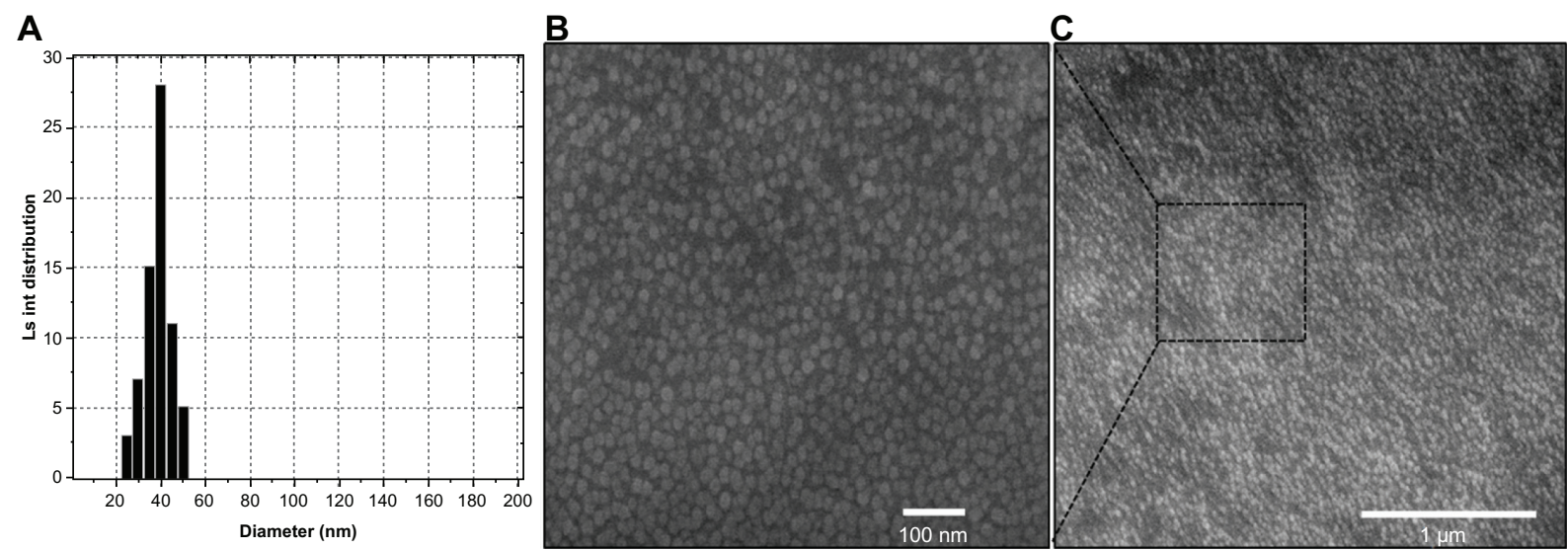

Figure 2 Size analysis of permeation-enhanced dual drug-loaded nanoparticles. (A) Particle size distribution of nanoparticles with 4.5 wt\% oleic acid had a uniform size range, as measured by dynamic light scattering. (B and $\mathbf{C}$ ) Field emission scanning electron microscopic images of nanoparticles with different magnification. Abbreviation: Ls int, light scattered intensity. 
freeze-dried nanoparticles recovered were suspended in $50 \mathrm{~mL}$ of dimethyl sulfoxide, mixed together for 1 hour, and finally subjected to ultracentrifugation at 20,000 rpm and $25^{\circ} \mathrm{C}$ for 30 minutes. The amount of drug present in the supernatant was analyzed by ultraviolet-visible spectroscopy at $282 \mathrm{~nm}$ and $374 \mathrm{~nm}$. A standard calibration curve was constructed using different concentrations of drug versus maximum absorbance. The maximum absorbance for supernatants of the drug-loaded polymeric particles was used for drug quantification. The entrapment efficiency of the drug-loaded nanoparticles was analyzed from the spectral absorbance areas of the supernatants. A standard calibration curve was constructed using different concentrations of drug (0.01-12.5 $\mu \mathrm{g}$ ) versus maximum absorbance (see Figure S2). The correlation coefficient for the linear range of the standard curve for voglibose was found to be 0.9997 and 0.999 . The nanoparticles containing oleic acid showed high encapsulation efficiency for quercetin and voglibose, reaching up to $92 \%$ and $86 \%$, respectively (Table 2 ). High encapsulation efficiency was achieved because of affinity between the positively and negatively charged groups on the surface of the nanoparticles. The initial drug concentration also had a significant effect on encapsulation efficiency.

\section{Preparation of film-forming gel and film}

Figure 3 shows the mass area morphology of film samples with uniform structure and shape (S1, S2, S3, S4, and blank). It displays the sequential changes in solid film formation from the cross-linked gels of varying composition compared with the blank film. EDC was used as a cross-linking agent because it could be easily washed off with aqueous solution after cross-linking; moreover, it is not incorporated with the targeted molecules. EDC forms a peptide bond between the primary amine and carboxyl groups of the polymer. The film forms a smooth surface which could reduce the risk of wounding or being rubbed off during dressing, and thereby improves upon traditional dressings. It is believed that the film can maintain satisfactory dressing performance on skin even after a prolonged period of time. These results indicate that such a scaffold film could have important physicochemical properties which could be suitable for long-term dressing and with enough strength to act as a drug carrier.

\section{Characterization of composite films}

This analysis was carried out in the region of $4000-400 \mathrm{~cm}^{-1}$ to confirm the chemical stability of the samples prepared. The initial FT-IR spectra of the dual drug-loaded nanoparticles using oleic acid (4.5 wt\%) are shown in Figure 4. The characteristic $\mathrm{PLGA}$ peaks for $-\mathrm{C}=\mathrm{O}$ and $-\mathrm{C}-\mathrm{O}$ stretching $\left(1618.3 \mathrm{~cm}^{-1}\right.$ and $\left.1108.1 \mathrm{~cm}^{-1}\right)$ appeared in the drug-loaded nanoparticles. Bands at $1676 \mathrm{~cm}^{-1}$ and $1601 \mathrm{~cm}^{-1}$ reflect amides I and II of PLGA. Peaks characteristic of quercetin were found in the range of $1100-1600 \mathrm{~cm}^{-1}$, and of hydroxyl bending at $1200-1400 \mathrm{~cm}^{-1}$; these were observed for the PLGA-quercetin-voglibose-oleic acid sample but were absent for the sample of PLGA alone. Further, the oleyl chain spectrum showed strong absorption bands at $2852 \mathrm{~cm}^{-1}$ and $2923 \mathrm{~cm}^{-1}$, arising from symmetric and asymmetric $\mathrm{C}-\mathrm{H}$ stretching of oleic acid, respectively, in the nanoparticles. These oleic acid stretching peaks were markedly decreased after molecule exchange, with new bands appearing at $1110 \mathrm{~cm}^{-1}$ and $1635 \mathrm{~cm}^{-1}$, possibly indicative of $\mathrm{C}-\mathrm{O}-\mathrm{C}$ stretching in PLGA. The PLGA nanoparticles did not show changes with respect to the PLGA polymer alone, indicating no interactions between the drugs and the nanoparticles. The encapsulation procedure did not alter the characteristics of the polymer or the drugs.

The polymer-based composite films prepared using different ratios of polyvinyl alcohol, sodium alginate, polyethylene glycol-600, hyaluronic acid, xylitol, and drug-loaded nanoparticles were also analyzed using FT-IR, the results of which are shown in Figure 5. Noncross-linked (blank) and cross-linked (S1, S2, S3, and S4 drug-loaded nanoparticle) films were characterized across a range of vibration frequencies (800-1800 $\mathrm{cm}^{-1}$ ) to ensure successful peptide bond formation. Peaks for two amide bonds were observed at $1645 \mathrm{~cm}^{-1}$ (amide I) due to $-\mathrm{C}=\mathrm{O}$ stretching vibrations of the natural polymer, and at $1541 \mathrm{~cm}^{-1}$ (amide II) due to $-\mathrm{C}(\mathrm{O})$ stretching of aliphatic amides and in plane $\mathrm{N}-\mathrm{H}$ bending mode. The amide III band was assigned at $1245 \mathrm{~cm}^{-1}$ for complex vibration mode. The films showed stronger intensity

Table 2 Percentage of nanoparticle recovery, drug content, entrapment, and wastage for nanoparticle preparation

\begin{tabular}{lllrr}
\hline $\begin{array}{l}\text { Drug-loaded } \\
\text { nanoparticle }\end{array}$ & $\begin{array}{l}\text { Nanoparticle } \\
\text { recovery (\%) }\end{array}$ & $\begin{array}{l}\text { Drug content } \\
(\%, w / w)\end{array}$ & \multicolumn{1}{c}{$\begin{array}{l}\text { Drug } \\
\text { entrapment (\%) }\end{array}$} & $\begin{array}{r}\text { Drug } \\
\text { wastage (\%) }\end{array}$ \\
\hline Voglibose & $86.45 \pm 0.006$ & $2.65 \pm 0.190$ & $84.0 \pm 0.054$ & $16.0 \pm 0.059$ \\
Quercetin & $92.12 \pm 0.010$ & $2.57 \pm 0.083$ & $86.64 \pm 0.164$ & $13.36 \pm 0.163$ \\
\hline
\end{tabular}

Notes: Mean \pm standard deviation, $\mathrm{n}=3$. 


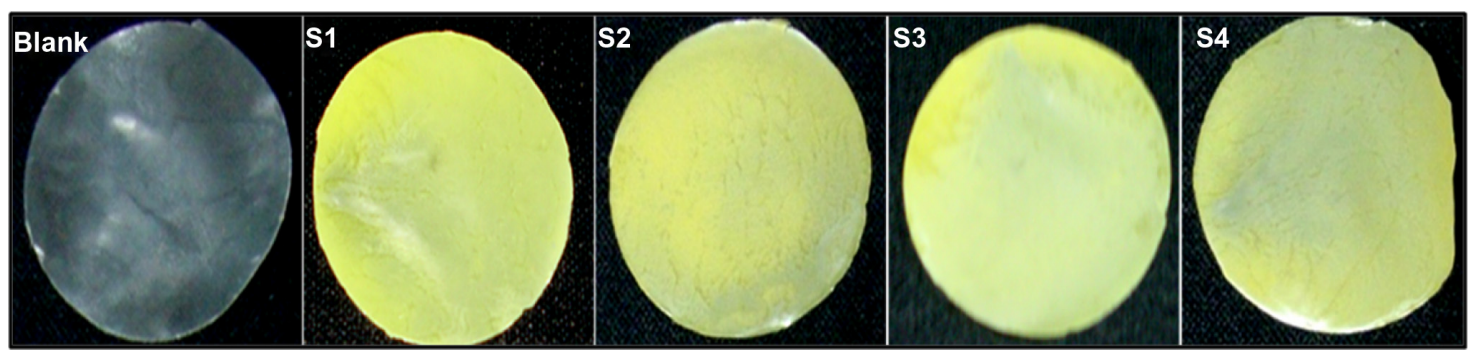

Figure 3 Mass area morphological features of films with uniform drug distribution.

Note: Surface and color differences were observed on films containing nanoparticles with various polymer compositions (SI, S2, S3, and S4) and blank film.

of the characteristic bands for the nanoparticles, suggesting successful incorporation of nanoparticles in the films. The spectrum ranges of $1050-1100 \mathrm{~cm}^{-1}$ and $2700-2950 \mathrm{~cm}^{-1}$ existed in both the drug-loaded nanoparticles and films (peaks for $\mathrm{COOH}$ and $\mathrm{OH}$ groups in the polymer film disappeared after cross-linking). The drug-loaded nanoparticles alone showed core peaks at wave numbers of $1050-1250 \mathrm{~cm}^{-1}$, and the same was noted in the S1, S2, S3, and S4 films, indicating there was no interaction between the drugs and the polymers. Moreover, no extra chemical bonding was observed, suggesting that EDC was not involved in the chemical interactions.

The thermal properties of the films were investigated using thermogravimetric analysis and are shown in Figure 6. Weight loss in the noncross-linked films (blank) was compared with that in the cross-linked films (S1, S2, S3, S4) by heating at a rate of $5^{\circ} \mathrm{C}$ per minute across a temperature range from $20^{\circ} \mathrm{C}$ to $100^{\circ} \mathrm{C}$, in a nitrogen atmosphere. The small amount of continuous weight loss is observed in noncross-linked film from initial temperature onwards and the overall weight loss observed more than $7 \%$. The weight loss observed was due to breakdown of the carboxyl molecules present in the polymers. However, weight loss from the cross-linked film was less than $5 \%$, and also showed an increment when the ratio of polyvinyl alcohol increased. The S1, S2, S3, and S4 samples show weight loss due to the decreased sodium alginate content and increased polyvinyl alcohol content (Table 1).

Figure S3 (see supplementary information) shows an $\mathrm{X}$-ray diffraction analysis of crystalline properties before and after cross-linking of the integrated film in the range of $10-90^{\circ}$ with a scan interval of 0.02 seconds. The noncrosslinked (blank) film shows crystalline peaks at $19^{\circ}$. The diffraction spectrum is similar to that of the drug-loaded nanoparticles. Principal diffraction peaks at $2 \theta=19^{\circ}$ were

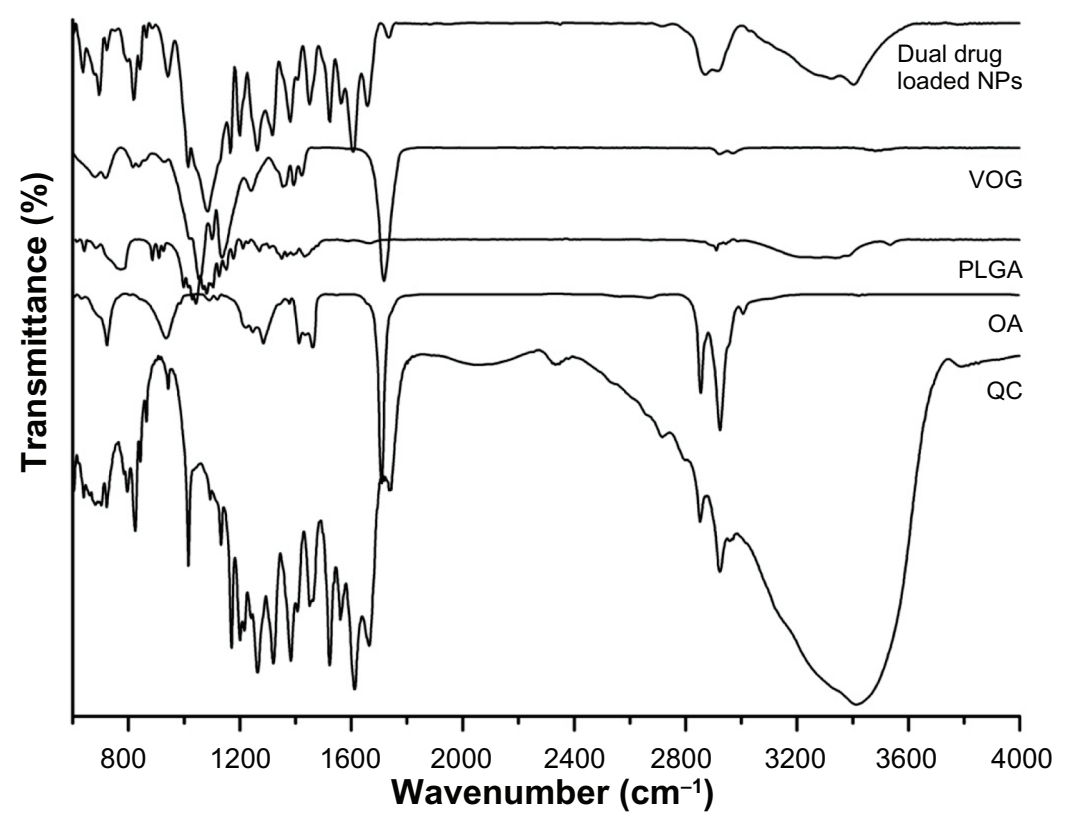

Figure 4 Fourier-transform infrared spectra of drugs, polymers, and drug-loaded nanoparticles. Abbreviations: NPs, nanoparticles; VOG, voglibose; OA, oleic acid; QC, quercetin; PLGA, poly(lactic-co-glycolic acid). 


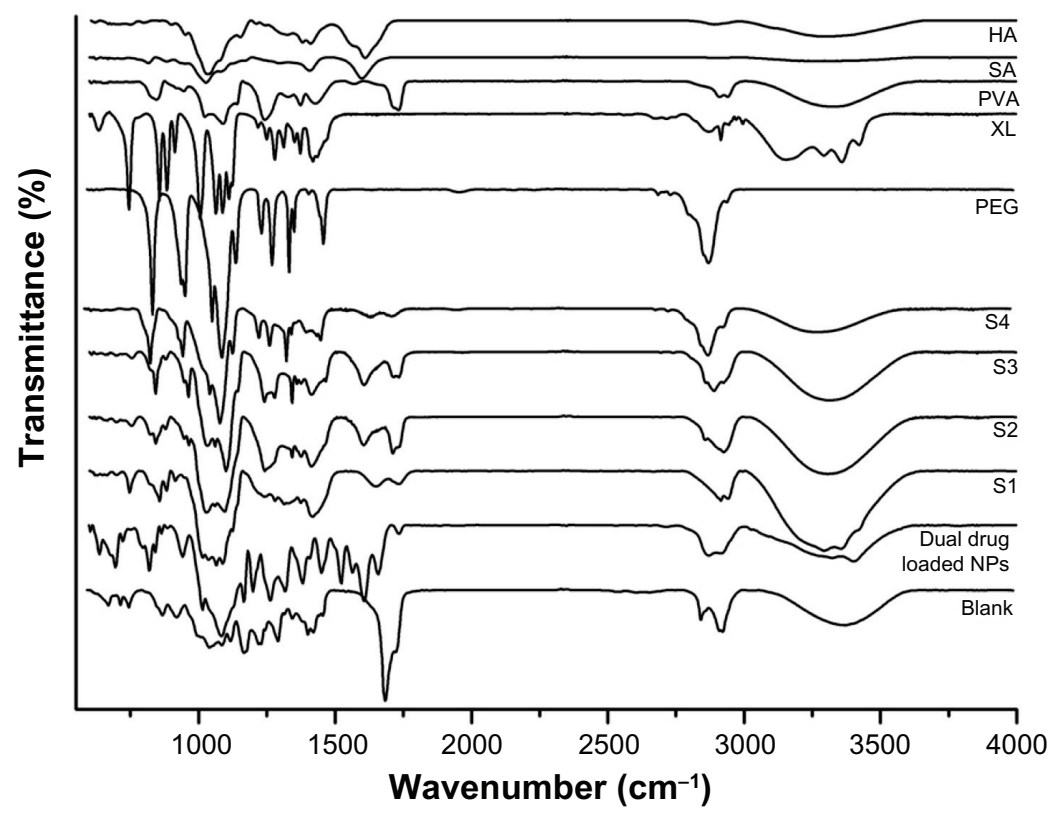

Figure 5. Fourier-transform infrared spectra of polymers used in film formation, drug-loaded nanoparticles, and nanoparticle-incorporated film with various compositions (SI, S2, S3, and S4).

Abbreviations: HA, hyaluronic acid; SA, sodium alginate; PVA, polyvinyl alcohol; XL, xylitol; PEG, polyethylene glycol; NPs, nanoparticles.

noticed for all the compositions, but the noncross-linked film showed a higher intensity peak than the cross-linked film due to crystalline regions in the polymers which resulted from strong hydrogen bonding between the hydroxyl groups. ${ }^{43}$ All the peak intensities of the drug-loaded films incorporating nanoparticles decreased steadily with increasing sodium alginate ratios (S1, S2, S3, and S4). An increase in the polyvinyl alcohol ratio increased these crystalline regions, ${ }^{50}$ indicating that sodium alginate suppresses the crystalline region of polyvinyl alcohol. Drugs showing a crystalline state in the nanoparticle composites were not detected in the S1-S4 films, indicating that the amorphous characteristics of sodium alginate diminished the crystallinity of the nanoparticles.

\section{Morphology and topography}

Figure S4A (see supplementary information, S1, S2, S3, S4, and blank) shows optical microscopic images for the films. It is noteworthy that the surface of the blank has a uniform

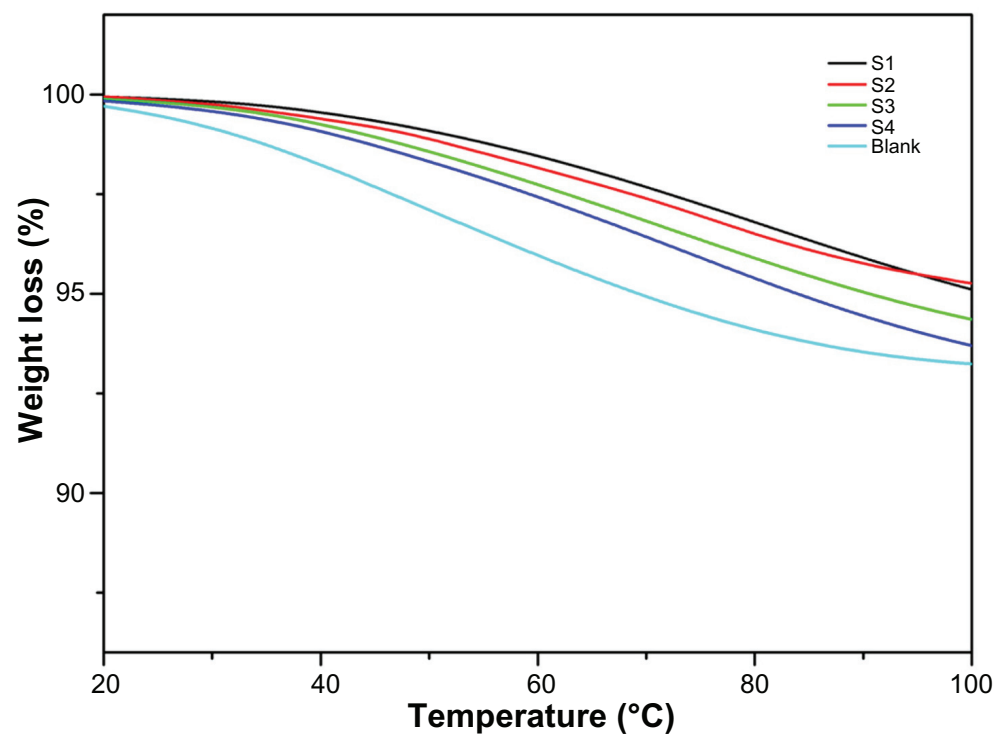

Figure $\mathbf{6}$ Thermogravimetric analysis curves for integrated scaffold films.

Note: The weight reduction for noncross-linked (blank) film and cross-linked films (SI, S2, S3, and S4). 
smooth surface without coarse particles. Aggregation of less dense, spherical-shaped, larger particles is seen in the S1 sample. Particle aggregation started to decrease as a function of change in the polymer concentration, leading to dense packing of particles in the scaffold network of the S2 and S3 films. Finally, highly dense and self-integrated scaffold film containing uniform particles can be seen in the S4 sample.

The smooth surface of the spherical drug-loaded nanoparticle-incorporated film was confirmed using field emission scanning electron microscopy and bioatomic force microscopy. The morphology of the drug-loaded nanoparticles can be clearly observed in Figure 2B. The images show that nanoparticles with a smooth surface and a homogeneous size were formed. Figure 2B demonstrates optimized and stabilized nanoparticles. The formulation containing oleic acid $4.5 \mathrm{wt} \%$ showed no aggregation or separation. Hence, this preparation was used for further studies. The morphology of the integrated scaffold films is shown in Figure 7. Micrographs of the blank and S1, S2, S3, and S4 scaffold network structures reveal that different polymer ratios influenced the surface properties of each sample. Of all the samples, S4 displayed the most homogenously dispersed scaffold film with the smoothest surface. Addition of a crosslinking agent significantly affected the surface properties of the film. The blank film shows a blended polymer scaffold with a homogeneous structure. The S1, S2, and S3 images do not show nanoparticles on the surface of the scaffolds, but all the nanoparticles are incorporated into the films. Moreover, S3 reveals a uniform network structure for the scaffolds. Finally, S4 shows uniform embedding of nanoparticles on the surface of the film. The blank film shows a clear homogeneous ribbon-like structure, whereas cross-linking enhances the network of the polymer-blended scaffold structures in the film, which varied according to concentration ratio. The nanoparticles formed can be self-integrated when different ratios of polyvinyl alcohol and sodium alginate are used in the solvent casting process. ${ }^{39}$ At low concentration of sodium alginate and high polyvinyl alcohol concentration, the nanoparticles significantly arranged in a regular network within the films. Moreover, the nanoparticles incorporated into the scaffold can improve the capacity for mechanical strength, smooth surface, and film formation. The uniform networklike structure of the nanoparticle-incorporated scaffold films was confirmed further by bioatomic force microscopy.

\section{Atomic force microscopy}

The surface topography and roughness of the integrated films before and after application were investigated using bioatomic force microscopy. Topography reveals that the surface roughness of the film increased with decreasing polyvinyl alcohol concentrations ( $\mathrm{S} 1, \mathrm{~S} 2, \mathrm{~S} 3$, and S4, respectively). Figure 8 shows the atomic force microscopic images of the films, which are consistent with those obtained by field emission scanning electron microscopy. The blank film shows a regular surface without nanoparticles. S1, S2, and S3 have few nanoparticles on the surface of the scaffolds, but all are incorporated into the films. S4 shows uniform incorporation of nanoparticles into the film. Incorporation of nanoparticles into the film was further confirmed after a release study. Root mean square values for roughness were calculated in the dry state before and after exposure to the release studies of the films and summarized in Table 3, the standard deviation of root mean square roughness was $\pm 0.07-0.22 \mathrm{~nm}$. The surface roughness of the films increased with decreasing polyvinyl alcohol and sodium alginate concentrations, as a result of high diffusion capacity. Inadequate availability of diffusion sites due to lower sodium alginate content may lead to reversal of the surface charge, and the roughness value is higher before application. The S1 sample with a low polyvinyl alcohol content showed high roughness of $18.0 \mathrm{~nm}$, whereas the S4 sample with a high polyvinyl alcohol concentration showed a smooth surface with a lower roughness value of $5.2 \mathrm{~nm}$. An opposite trend was noticed for sodium alginate (S1), high concentrations of which decreased surface roughness, while low concentrations increased surface roughness. The atomic force microscopic images (Figure 8) are noteworthy with regard to surface roughness. Importantly, roughness decreased dramatically on the noncross-linked film as a result of gaining amine and carboxylic acid groups by the films. During degradation process, breaking down of the chemical bonds in the crosslinked film causes the reduction in surface roughness. This observation suggests that the film has undergone surface erosion. ${ }^{51}$ These findings indicate that this smooth-surfaced composite polymer film might be safe for treatment of skin wounds.

\section{Effects of film swelling rates}

The effects of environmental conditions on swelling of the integrated films were determined under physiological conditions using different temperatures, $\mathrm{pH}$ levels, and time intervals. Figure 9A indicates that the films displayed $\mathrm{pH}$ sensitivity in their swelling behavior and kept changing until an equilibrium was reached. Low pH (5.5) decreased the swelling rate and minimized it further upon increasing the $\mathrm{pH}$ to 6.5 , while a high $\mathrm{pH}$ (7.5) increased the swelling rate. The highest swelling rate noticed for $\mathrm{S} 4$ at $\mathrm{pH} 6.5$ was 

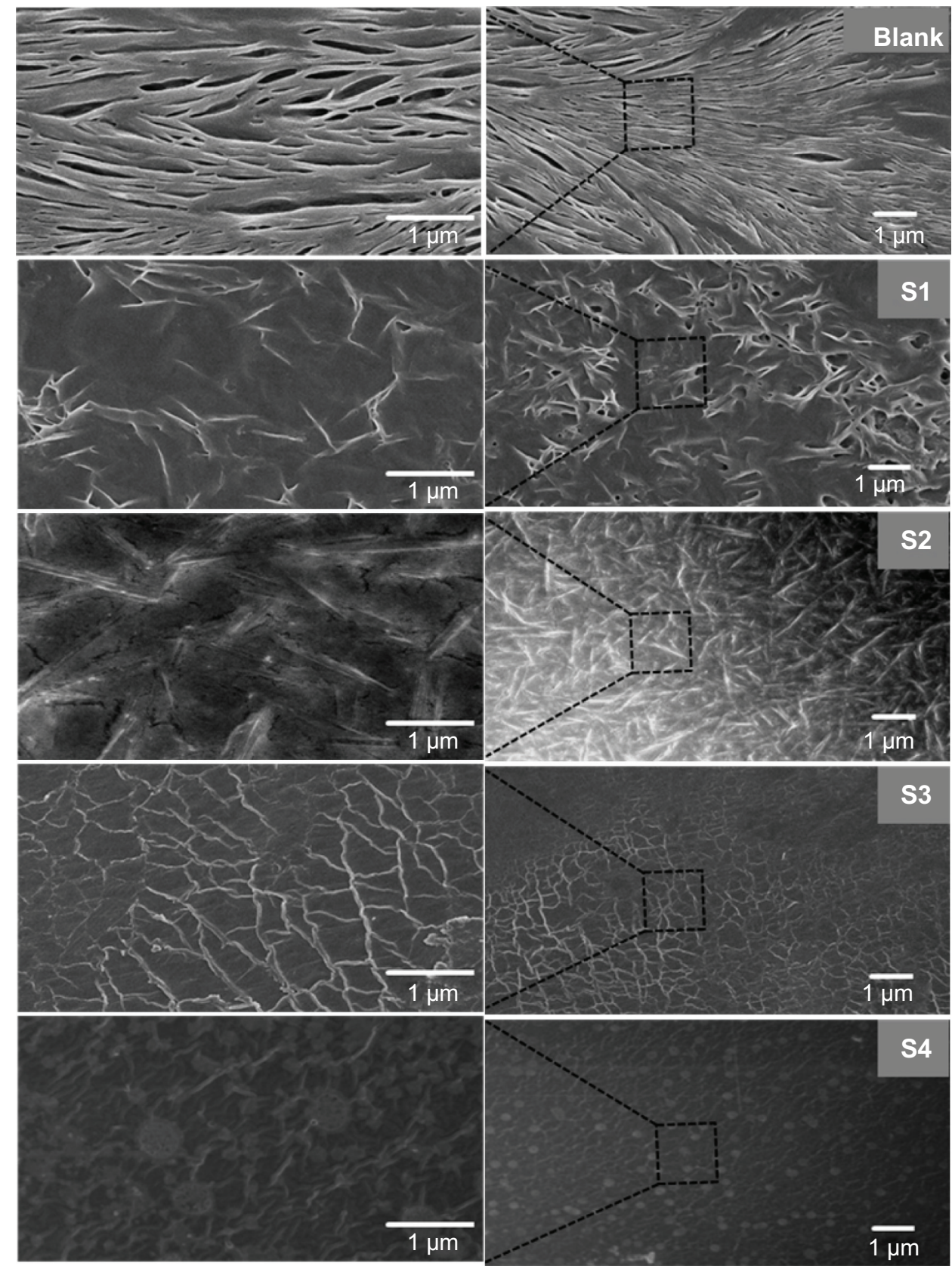

Figure 7 Field emission scanning electron microscopic images for the nanoparticle-incorporated polymer composite on self-integrated scaffold films with and without crosslinking (blank).

Notes: Left side is a representative magnified field emission scanning electron microscopic image of the right side image of an integrated scaffold film. Blank film shows blended polymer scaffold structure. S1, S2, and S3 have no nanoparticles on the surface of the scaffolds, but all the nanoparticles are incorporated into the films. S3 shows scaffolds, with a uniform network structure and S4 shows a regular nanoparticle incorporation into scaffolds, forming a uniform network structure.

due to an increase in ionic swelling pressure. The formation of film networks containing oppositely charged carboxyl and amino groups could be changed by varying the $\mathrm{pH}$. Also, the dominant charges in the film could be controlled in the presence of amino groups and carboxyl groups by modifying low and high $\mathrm{pH}$ levels.

Figure 9B shows the thermoresponsive swelling film rates. An elevated swelling rate was noticed with a temperature increase from $27^{\circ} \mathrm{C}$ to $47^{\circ} \mathrm{C}$ in the surrounding medium. The swelling rate remained constant below $27^{\circ} \mathrm{C}$, but the film collapsed above $47^{\circ} \mathrm{C}$ because of the water molecules associated with the film components being liberated at an increased temperature. Because the blank contained more free amino groups, it could easily form hydrogen bonds with water molecules, thereby showing an increased swelling rate leading to collapse. Swelling of the other films 
A
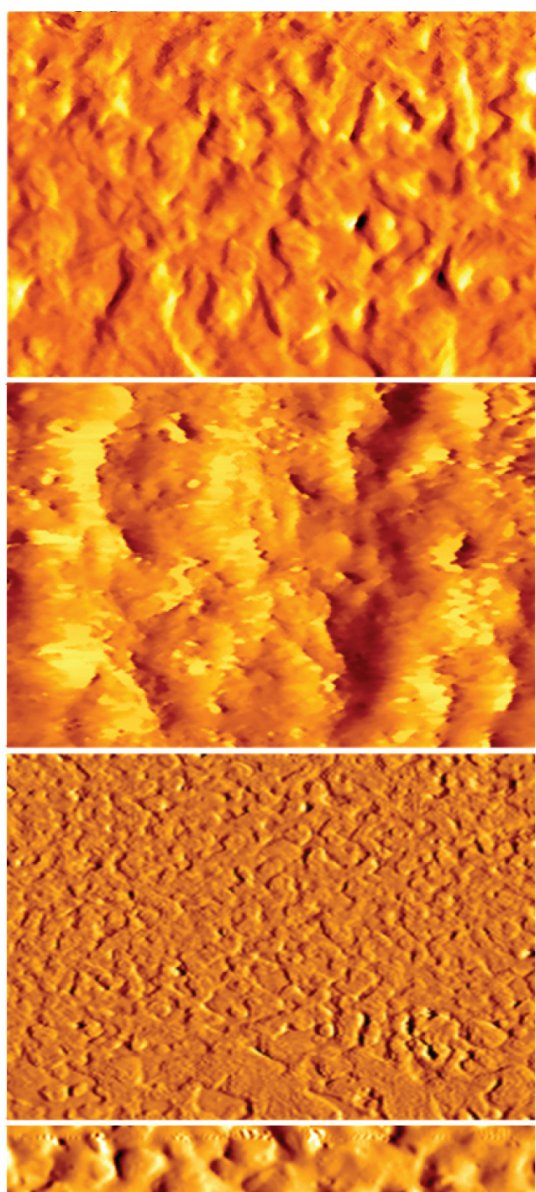

B
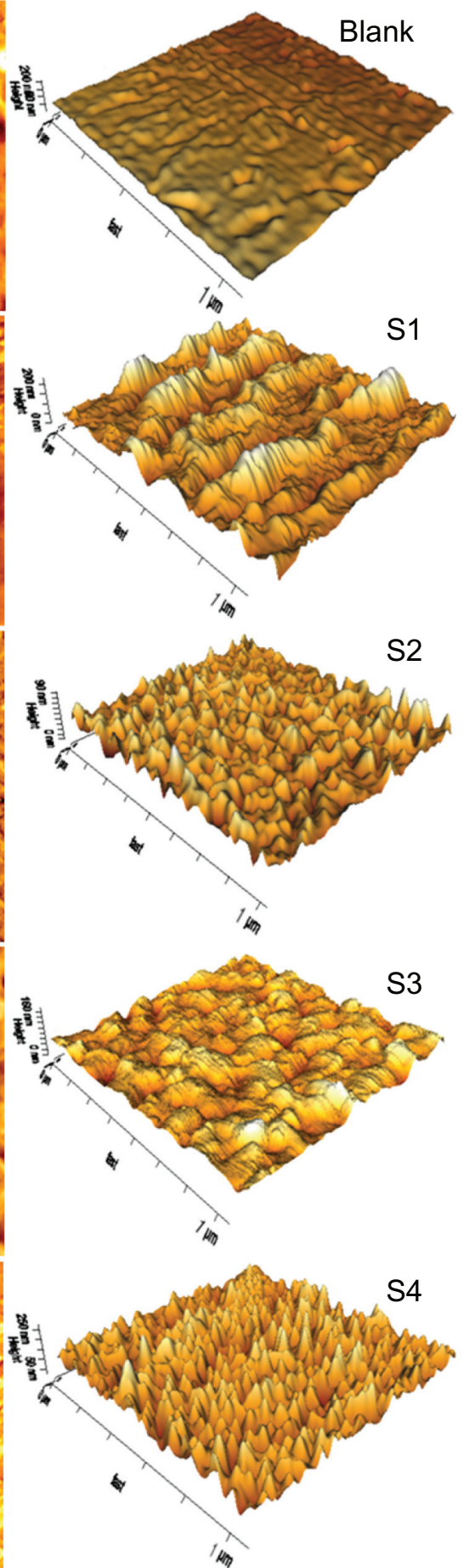

Figure 8 Topographical atomic force microscopy images of nanoparticle-incorporated polymer composite scaffold films with and without cross-linking (blank) in the dry state before the drug release study. (A) Atomic force microscopy lock-in amplitude image of integrated scaffold films. (B) Three-dimensional atomic force microscopy height images of A. Note: Scan size I $\times$ I $\mu \mathrm{m}$.

could be controlled due to the presence of EDC cross-linking. Swelling of the cross-linked film did not lead to collapse at high temperatures. However, polymers with a cross-linking agent showed decreased water solubility as the temperature increased. This demonstrates that the polymer used for film fabrication has temperature-dependent swelling properties. These films are made of polymer chains that either possess moderately hydrophobic groups or contain a mixture of hydrophilic and hydrophobic segments. At lower temperatures, hydrogen bonding between the hydrophilic segments of 
Table 3 Roughness of films before and after application

\begin{tabular}{lll}
\hline Samples & \multicolumn{2}{l}{ Root mean square roughness } \\
\cline { 2 - 3 } & Before & After \\
\hline Blank & 26 & 18.3 \\
SI & 18 & 12.06 \\
S2 & 12.6 & 11.1 \\
S3 & 9.8 & 5.8 \\
S4 & 5.2 & 3.3 \\
\hline
\end{tabular}

the polymer chain and water molecules dominates, leading to enhanced dissolution in water. As the temperature increases, hydrophobic interactions among the hydrophobic segments become stronger while hydrogen bonding becomes weaker. ${ }^{52}$ Other factors influencing swelling of the films could include the hydrophilicity of the polymer chains, the blending ratio, and the density of the network.

\section{Ex vivo drug release study}

Release of quercetin and voglibose from the nanoparticleincorporated hybrid film into the skin is shown in Figure 10. All the films exhibited a permeation-enhanced drug release rate as compared with the blank film, and showed significant drug release over a period of 72 hours, after which the skin properties collapsed. The same release pattern was observed for both quercetin and voglibose, but the cumulative release of quercetin was much higher than that of voglibose. Of all the films, S4 showed the best controlled drug release rates of $92 \%$ and $50 \%$ for quercetin and voglibose, respectively. The results

B
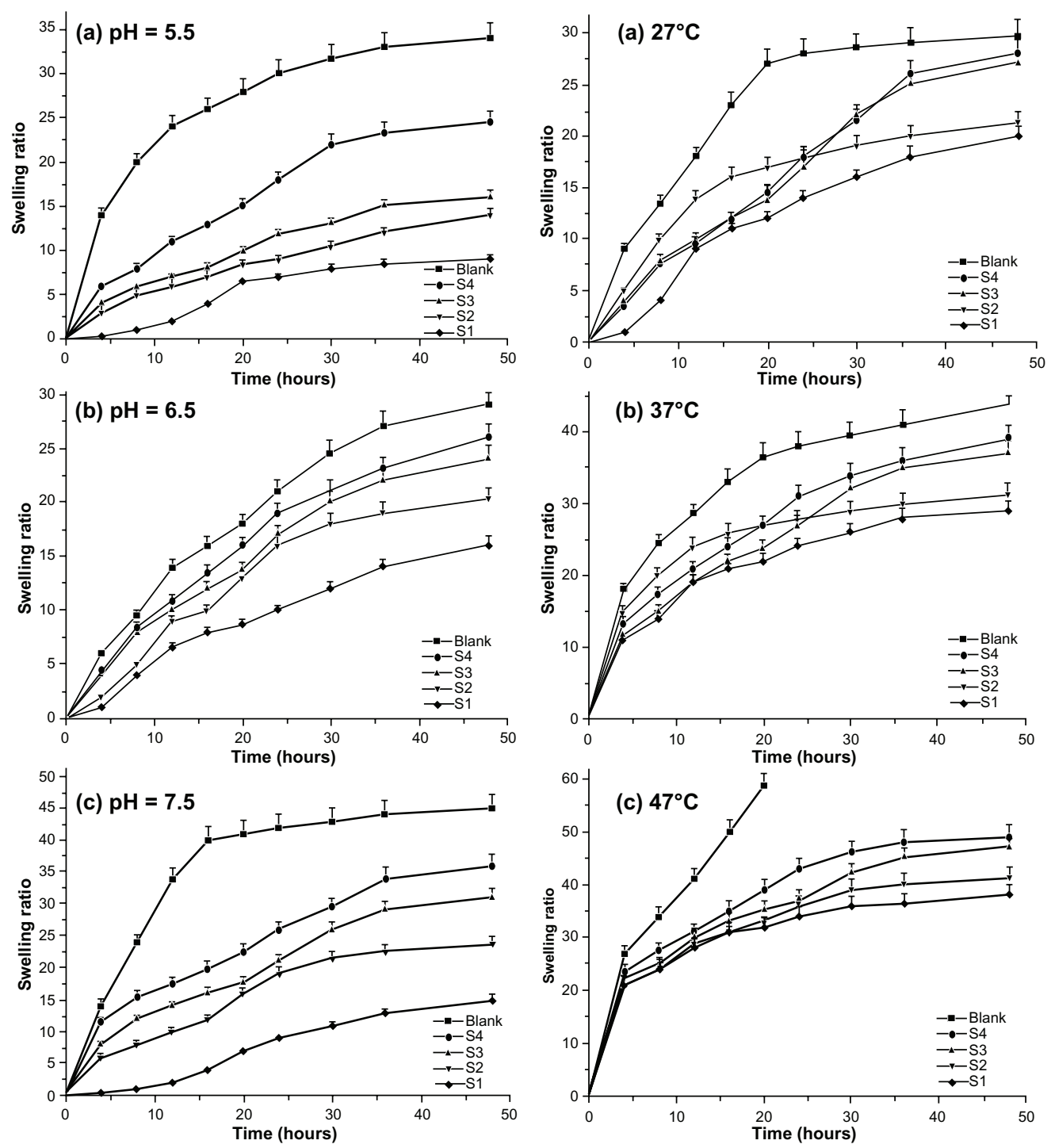

Figure 9 Swelling properties of various types of scaffold films. Swelling ratio of films in solution with different $\mathrm{pH}$ and temperatures. (A) Swelling ratios of films in solutions with different $\mathrm{pH}$ values, ie, (a) 5.5, (b) 6.5 , and (c) 7.5. (B) Swelling ratios of films in solutions with different temperatures, ie, (a) $27^{\circ} \mathrm{C}$, (b) $37^{\circ} \mathrm{C}$, and (c) $47^{\circ} \mathrm{C}$. 
A

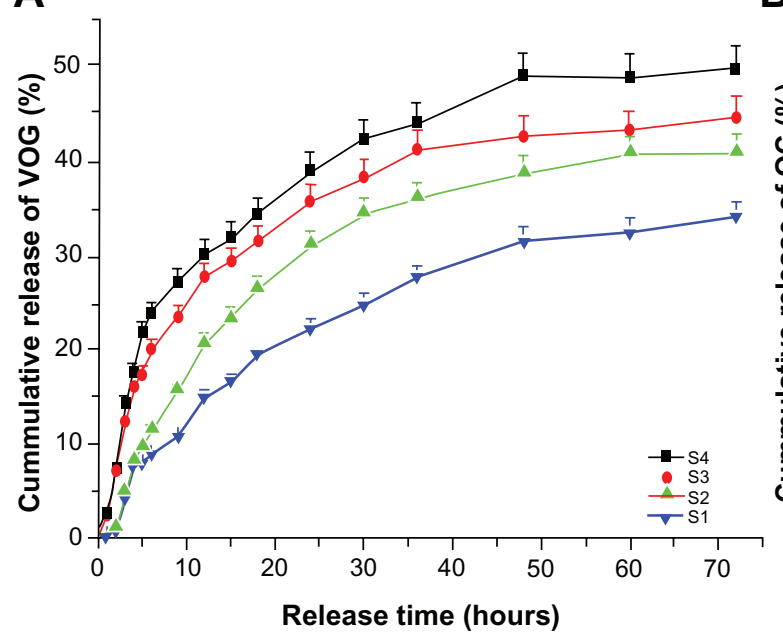

B

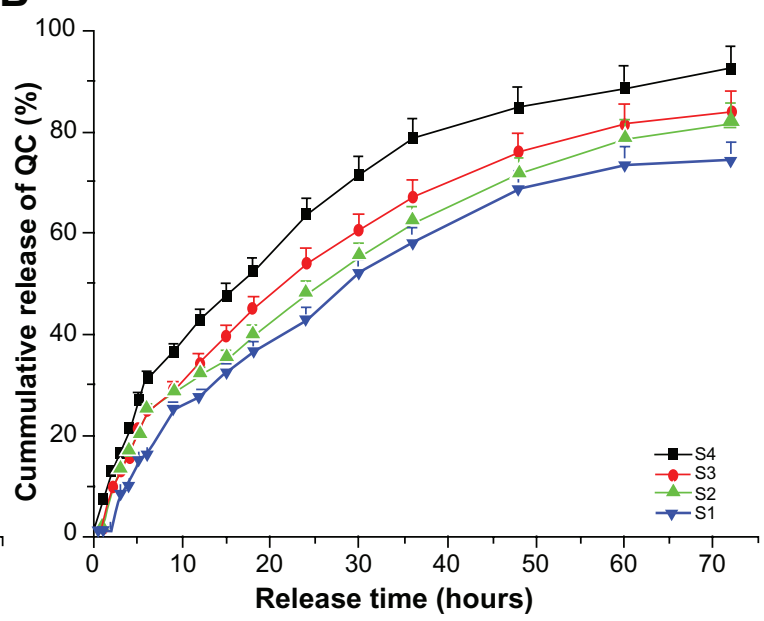

Figure 10 Release profiles of quercetin and voglibose from nanoparticle-incorporated polymer composite film. (A) Release profiles of quercetin from all films (SI, S2, S3, and S4). (B) Release profiles of voglibose from all films (SI, S2, S3, and S4).

Abbreviations: VOG, voglibose; QC, quercetin.

indicate that cumulative release of quercetin and voglibose from the S4 film was more significant, and contained a suitable composite ratio for enhanced drug diffusion. It is well known that glycol creates a large free volume within a hybrid film, leading to increased drug diffusion. ${ }^{53}$ This can be seen in the $\mathrm{S} 4$ hybrid film. The experiment referred to above showed that a suitable quantity of sodium alginate can enhance the amorphous region of the film effectively and, accordingly, enhance drug diffusion. On the other hand, the excessive self-crosslinked reaction of these composite polymers made the hybrid film more closely constrained, and also made drug diffusion more difficult. Moreover, the S4 hybrid film with the composite polymers described showed significant release of quercetin and voglibose, but the other hybrid films showed limited drug release. Also, combined incorporation of an antioxidant and an antidiabetic agent improved the performance of the composite polymers. The use of transdermal delivery systems such as liposomes, transfersomes, ethosomes, and niosomes is still limited because of stability issues. Skin permeability relative to different shapes and sizes of nanoparticles has also been studied. ${ }^{54}$ In relation to the above study, the present research has shown that better skin penetration of nanoparticles from the film is due to very tiny spherical shape of particles which is achieved in the presence of chemical permeation enhancer. Formulation of a nanoparticle-incorporated scaffold film was achieved to develop an effective transdermal delivery system for antioxidant and hypoglycemic compounds, such as quercetin and voglibose. The films contain very tiny nanoparticles with enhanced permeation ability when comparing their transdermal delivery efficiency with that of conventional vehicles, including microemulsions containing quercetin, quercetin-loaded lecithin-chitosan nanoparticles, ${ }^{55}$ and colloidal polymeric particles. ${ }^{54}$ The skin penetration results showed that permeation-enhanced dual drug-loaded nanoparticles incorporated in the scaffold film improved the rate of controlled drug release.

\section{Morphologies of hybrid scaffold films after drug release}

Twenty-four hours into the drug release study, morphological changes were observed for the films on light microscopy, field emission scanning electron microscopy, and bioatomic force microscopy. Light microscopic images (Figure S4A and B, see supplementary information) show the morphology of blank and hybrid films before and after the release studies. As observed, all the films had a uniform size and shape before the release studies (Figure S4, see supplementary information) whereas after the release studies there were significant bulging and irregular shapes with protrusions observed on the surface of the films (Figure S4B, see supplementary information). Field emission scanning electron microscopic images (Figure S4C, see supplementary information) show the morphology of swelling structures and projection shapes on the blank, S1, S2, S3, and S4 films. The scanning electron microscopic images further confirm controlled drug release from the cross-linked hybrid films, indicated by changes in the intermolecular network of polymer composites. Bioatomic force microscopic images (Figure S5, see supplementary information) show the morphology of the swelling structures with a smooth surface and with projections from the blank 


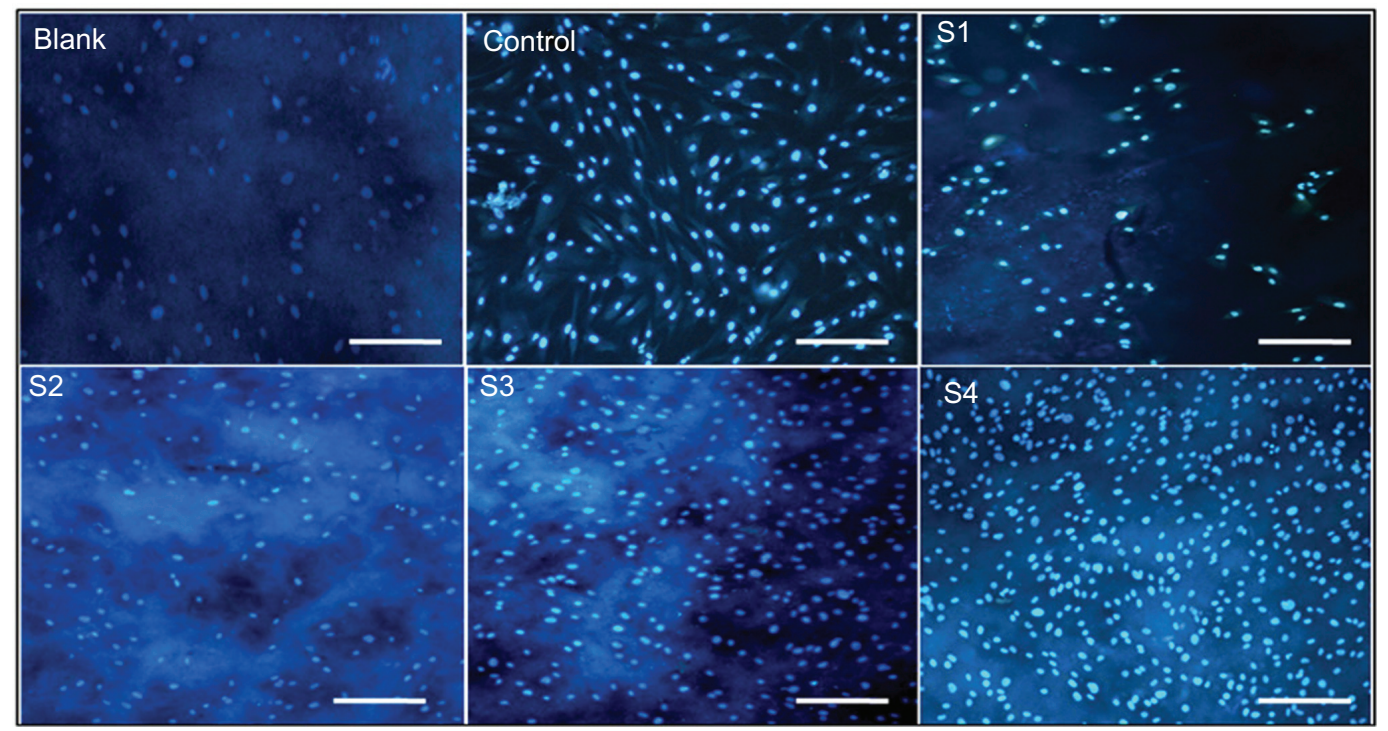

Figure I I Fluorescence photomicrograph of control fibroblasts, fibroblasts treated with blank, SI, S2, S3, S4 films and control (without film) for 3 days. Note: Scale bar, $20 \mu \mathrm{m}$ at $50 \times$ magnification.

and S1, S2, S3, S4 films, respectively. The projections formed on the surface of the nanoparticle-incorporated scaffold films ensure the release of nanoparticles. The root mean square roughness values for the films were calculated before and after exposure in the release studies, and are summarized in Table 3. After exposure, all the films showed decreased surface roughness. Notably, as discussed above, roughness decreased dramatically in the noncross-linked film due to gaining of amine and carboxylic acid groups in the films.

\section{In vitro biocompatibility studies of self-integrated scaffold films}

The biocompatibility of the nanoparticle-incorporated scaffold films was evaluated using human fibroblasts (FY11 cells)

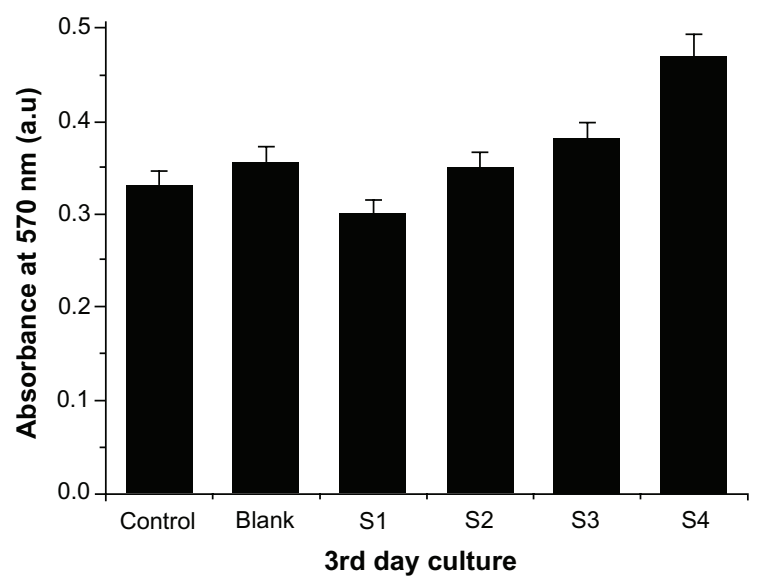

Figure 12 (3-(4,5-Dimethylthiazol-2-yl)-2,5-diphenyltetrazolium bromide) assays of integrated scaffold films with human fibroblasts treated for 3 days. cultured on the blank and hybrid film surfaces for 3 days Nuclear staining with DAPI was carried out on the third day of FY11 cell culture, and fluorescence images were then taken (Figure 11). The cells were more compatible and well adhered on the S4 film surface. Cell viability was estimated by measuring mitochondrial activity in the cell using an MTT assay kit. Figure 12 shows the MTT assay of films with FY11 cells, indicating that absorbance decreased with increasing concentrations of polyvinyl alcohol sequentially from S1, S2, S3, to S4 (0.3, 0.35, 0.38, and 0.47), respectively. The absorbance of the blank, S1, and S2 film was lower than that of the S4 film, clearly indicating that nanoparticle-incorporated films with an appropriate polymer ratio (S4) are cell-friendly and biocompatible, so could serve as a good alternative carrier in various biomedical applications.

\section{Conclusion}

Drug-loaded nanoparticles in transdermal polymeric scaffold films were prepared utilizing a solvent evaporation/casting technique and an EDC cross-linking method to produce a transdermal drug-delivery system. The present work utilized modified fabrication methods for development of permeation-enhanced dual drug-loaded nanoparticles. A new type of hybrid-integrated scaffold film consisting of sodium alginate, polyvinyl alcohol, polyethylene glycol-600, hyaluronic acid, and xylitol was developed with unique properties for a transdermal delivery system. Morphological analysis of films using optical microscopy and scanning electron microscopy indicates that the size, shape, and surface texture of the films 
were optimized by altering the polymeric ratio. The swelling rate increased with polymer concentration, and cross-linking indicated that the films had improved mechanical strength and that the polymer used for film fabrication had temperaturedependent swelling properties. The FT-IR film spectra show no structural deformation of the polymer in the nanoparticles after EDC cross-linking. Thermogravimetric analysis shows less weight loss from the cross-linked films than from the noncross-linked films, confirming the increased thermal stability of the cross-linked film. X-ray diffraction results show that the films became more crystalline after each decrement in the ratio of alginate, with increased amorphous properties. Orbicular nanoparticles of relatively uniform size were seen embedded in the polymeric surface of the scaffold films using atomic force microscopy. The dual drug-loaded nanoparticleincorporated films (S4) showed the most favorable controlled drug release and improved drug permeation across the skin. The film demonstrated high cell compatibility with less toxicity and good cell adherence on the film surface. Scaffold films are biocompatible and biodegradable, and could potentially be used for dual drug delivery. This novel film fabrication method is flexible, simple, and cost-effective. The prepared film is suitable for incorporation of nanoparticles, biomolecules, and therapeutic agents, and opens up a range of biomedical applications, such as wound dressing, management of diabetes-related complications, and transdermal drug delivery, with better patient compliance. Research into fundamental drug-drug and drug-polymer interactions, as well as the physicochemical properties of the materials used, are ongoing in our laboratory.

\section{Acknowledgment}

This work was supported by the Gachon University Research Fund of 2012 (KWU 2011-R372).

\section{Disclosure}

The authors report no conflicts of interest in this work.

\section{References}

1. Ding H, Triggle CR. Endothelial cell dysfunction and the vascular complications associated with type 2 diabetes: assessing the health of the endothelium. Vasc Health Risk Manag. 2005;1(1):55-71.

2. Stocker R, Keaney JF Jr. Role of oxidative modifications in atherosclerosis. Physiol Rev. 2004;84(4):1381-1477.

3. Johansen JS, Harris AK, Rychly DJ, Ergul A. Oxidative stress and the use of antioxidants in diabetes: Linking basic science to clinical practice. Cardiovasc Diabetol. 2005;4(1):1475-2840.

4. Maritim AC, Sanders RA, Watkins JB 3rd. Diabetes, oxidative stress, and antioxidants: a review. J Biochem Mol Toxicol. 2003;17(1):24-38.

5. Nichols JA, Katiyar SK. Skin photoprotection by natural polyphenols: anti-inflammatory, anti-oxidant and DNA repair mechanisms. Arch Dermatol Res. 2010;302(2):71-83.
6. Bonina F, Lanza M, Montenegro L, et al. Flavonoids as potential protective agents against photo-oxidative skin damage. Int $J$ Pharm. 1996;145(1-2):87-94.

7. Esposito K, Nappo F, Marfella R, et al. Inflammatory cytokine concentrations are acutely increased by hyperglycemia in humans role of oxidative stress. Circulation. 2002;106(16):2067-2072.

8. Esposito K, Marfella R, Giugliano D. Stress hyperglycemia, inflammation, and cardiovascular events. Diabetes Care. 2003;26(5):1650-1651.

9. Heimberg H, Heremans Y, Jobin C, et al. Inhibition of cytokineinduced NF-kB activation by adenovirus-mediated expression of a NF-kB super-repressor prevents $\beta$-cell apoptosis. Diabetes. 2001; 50(10):2219-2224.

10. Lin HJ, Lu AY. Role of pharmacokinetics and metabolism in drug discovery and development. Pharmacol Rev. 1997;49(4):403-449.

11. Satoh N, Shimatsu A, Yamada K, et al. An alpha-glucosidase inhibitor, voglibose, reduces oxidative stress markers and soluble intercellular adhesion molecule 1 in obese type 2 diabetic patients. Metabolism. 2006;55(6):786-793.

12. Yan S, Xiaoqiang L, Shuiping L, Xiumei M, Ramakrishna S. Controlled release of dual drugs from emulsion electrospun nanofibrous mats. Colloids Surf B Biointerfaces. 2009;73(2):376-381.

13. Xu J, Jiao Y, Shao X, Zhou C. Controlled dual release of hydrophobic and hydrophilic drugs from electrospun poly(L-lactic acid) fiber mats loaded with chitosan microspheres. Mater Lett. 2011;65(17-18):2800-2803.

14. Li YQ, Zhou FC, Gao F, Bian JS, Shan F. Comparative evaluation of quercetin, isoquercetin and rutin as inhibitors of alpha-glucosidase. J Agric Food Chem. 2009;57(24):11463-11468.

15. Li M, Al-Jamal KT, Kostarelos K, Reineke J. Physiologically based pharmacokinetic modeling of nanoparticles. ACS Nano. 2010; 4(11):6303-6317.

16. Guzik TJ, Mussa S, Gastaldi D, et al. Mechanisms of increased vascular superoxide production in human diabetes mellitus: role of $\mathrm{NAD}(\mathrm{P}) \mathrm{H}$ oxidase and endothelial nitric oxide synthase. Circulation. 2002;105(14):1656-1662.

17. Ju-Ichi M. Chemical study of citrus plants in the search for cancer chemopreventive agents. Yakugaka Zasshi. 2005;125(3):231-254. Japanese.

18. Kolishetti N, Dhar S, Valencia PM, et al. Engineering of self-assembled nanoparticle platform for precisely controlled combination drug therapy. Proc Natl Acad Sci U S A. 2010;107(42):17939-17944.

19. Tang DW, Yu S, Ho YC, Mi FL, Kuo PL, Sung HW. Heparinized chitosan/poly(-glutamic acid) nanoparticles for multi-functional delivery of fibroblast growth factor and heparin. Biomaterials. 2010;31(35): 9320-9332.

20. Misra R, Sahoo SK. Co-formulation of doxorubicin and curcumin in poly(D,L-lactide-co-glycolide) nanoparticles suppresses the development of multidrug resistance in K562 cells. Mol Pharm. 2011; 8(3):852-866.

21. Song XR, Cai Z, Zheng Y, et al. Reversion of multidrug resistance by co-encapsulation of vincristine and verapamil in PLGA nanoparticles. Eur J Pharm Sci. 2009;37(3-4):300-305.

22. Mahapatro A, Singh DK. Biodegradable nanoparticles are excellent vehicle for site directed in-vivo delivery of drugs and vaccines. J Nanobiotechnology. 2011;9(1):55.

23. Amnuaikit C, Ikeuchi I, Ogawara K, Higaki K, Kimura T. Skin permeation of propranolol from polymeric film containing terpene enhancers for transdermal use. Int J Pharm. 2005;289(1-2):167-178.

24. Higaki K, Nakayama K, Suyama T, Amnuaikit C, Ogawara K, Kimura T. Enhancement of topical delivery of drugs via direct penetration by reducing blood flow rate in skin. Int J Pharm. 2005;288(2): 227-233.

25. Yerramsetty KM, Rachakonda VK, Neely BJ, Madihally SV, Gasem KAM. Effect of different enhancers on the transdermal permeation of insulin analog. Int J Pharm. 2010;398(1-2):83-92.

26. Vaddi HK, Ho PC, Chan YW, Chan SY. Terpenes in ethanol: haloperidol permeation and partition through human skin and stratum corneum changes. J Control Release. 2002;81(1-2):121-133. 
27. Kumar MN, Muzzarelli RA, Muzzarelli C, Sashiwa H, Domb AJ. Chitosan chemistry and pharmaceutical perspectives. Chem Rev. 2004;104(12):6017-6084.

28. Subbiah R, Veerapandian M, Yun KS. Nanoparticles: functionalization and multifunctional applications in biomedical sciences. Curr Med Chem. 2010;17(36):4559-4577.

29. Srinivasa PC, Ramesh MN, Tharanathan RN. Effect of plasticizers and fatty acids on mechanical and permeability characteristics of chitosan films. Food Hydrocolloid. 2007;21(7):1113-1122.

30. Zhang ML, Gong XH, Zhao YD, Zhang NM. Properties and biocompatibility of chitosan films modified by blending with PEG. Biomaterials. 2002;23(13):2641-2648.

31. Badet C, Furiga A, Thebaud N. Effect of xylitol on an in vitro model of oral biofilm. Oral Health Prev Dent. 2008;6(4):337-341.

32. Chandy T, Mooradian DL, Rao GH. Chitosan/polyethylene glycolalginate microcapsules for oral delivery of hirudin. J Appl Polym Sci. 1998;70(11):2143-2153.

33. Rajangam T, Paik HJ, An SSA. Development of fibrinogen microspheres as a biodegradable carrier for tissue engineering. Bio Chip J. 2011;5(2):175-183.

34. Bromberg LE, Ron ES. Temperature-responsive gels and thermogelling polymer matrices for protein and peptide delivery. Adv Drug Deliv Rev. 1998;31(3):197-221

35. Jain GK, Sharma AK, Agrawal SS. Transdermal controlled administration of verapamil -enhancement of skin permeability. Int J Pharm. 1996;130(2):169-177.

36. Ourique AF, Pohlmann AR, Guterres SS, Beck RCR. Tretinoin-loaded nanocapsules: preparation, physicochemical characterization and photostability study. Int J Pharm. 2008;352(1-2):1-4.

37. Liong M, Lu J, Kovochich M, et al. Multifunctional inorganic nanoparticles for imaging, targeting, and drug delivery. ACS Nano. 2008;2(5):889-896.

38. Comelles F, Sanchez-Leal J, Gonzalez JJ. Soybean oil microemulsions with oleic acid/glycols as cosurfactants. J Surfactants Deterg. 2005;8(3):257-262.

39. Kumari A, Yadav SK, Pakade YB, Singh B, Yadav SC. Development of biodegradable nanoparticles for delivery of quercetin. Colloids Surf B Biointerfaces. 2010;80(2):184-192.

40. Comelles F, Sanchez-Leal J, Gonzalez JJ. Influence of ionic surfactants on the formation of liquid crystals in oleic acid/glycol/water systems. J Surfactants Deterg. 2007;10(3):137-144.

41. Dhana LUM, Poovi G, Kishore N, Reddy PN. In-vitro characterization and in-vivo toxicity study of repaglinide loaded poly (methyl methacrylate) nanoparticles. Int J Pharm. 2010;396(1-2):194-203.
42. Mallikarjuna RN, Bagyalakshmi J, Ravi TK. Development and validation of UV-spectroscopic method for estimation of voglibose in bulk and tablets. J Chem Pharm Res. 2010;2(2):350-356.

43. Guo R, Du X, Zhang R, Deng L, Dong A, Zhang J. Bioadhesive film formed from a novel organic-inorganic hybrid gel for transdermal drug delivery system. Eur J Pharm Biopharm. 2011;79(3):574-583.

44. Cui F, He C, Yin L, et al. Nanoparticles incorporated in bilaminated films: a smart drug delivery system for oral formulations. Biomacromolecules. 2007;8(9):2845-2850.

45. Adhirajan N, Shanmugasundaram N, Babu M. Gelatin microspheres crosslinked with EDC as a drug delivery system for doxycycline: development and characterization. J Microencapsul. 2007;24(7):659-671.

46. Marimuthu M, Kim S, An J. Amphiphilic triblock copolymer and a microfluidic device for porous microfiber fabrication. Soft Matter. 2010;6(10):2200-2207.

47. Kumar TR, Shanmugasundaram N, Babu M. Biocompatible collagen scaffolds from a human amniotic membrane: physiochemical and in vitro culture characteristics. J Biomater Sci Polym Ed. 2003; 14(7):689-706.

48. Aryal S, Hu CM, Zhang L. Polymeric nanoparticles with precise ratiometric control over drug loading for combination therapy. Mol Pharm. 2011;8(4):1401-1407.

49. Bennet D, Kim S. A transdermal delivery system to enhance quercetin nanoparticle permeability. J Biomater Sci Polym E. (In press).

50. Gupta NV, Gowda DV, Balamuralidhara V, Mohammed Khan S. Formulation and evaluation of olanzapine matrix pellets for controlled release. DARU J Pharm Sci. 2011;19(4):249-256.

51. Fredin NJ, Zhang JT, Lynn DM. Surface analysis of erodible multilayered polyelectrolyte films: Nanometer-scale structure and erosion profiles. Langmuir. 2005;21(13):5803-5811.

52. Schild HG. Poly(N-isopropylacrylamide): experiment, theory and application. Prog Polym Sci. 1992;17(2):163-249.

53. Zhang J, Deng L, Zhao H, et al. Pressure-sensitive adhesive properties of poly(N-vinyl pyrrolidone)/D,L-lactic acid oligomer/glycerol/water blends for TDDS. J Biomater Sci Polym Ed. 2010;21(1):1-15.

54. Batheja P, Sheihet L, Kohn J, Singer AJ, Michniak-Kohn B. Topical drug delivery by a polymeric nanosphere gel: formulation optimization and in vitro and in vivo skin distribution studies. $J$ Control Release. 2011;149(2):159-167.

55. Tan Q, Liu W, Guo C, Zhai G. Preparation and evaluation of quercetin-loaded lecithin-chitosan nanoparticles for topical delivery. Int J Nanomedicine. 2011;6:1621-1630. 


\section{Supplementary information}

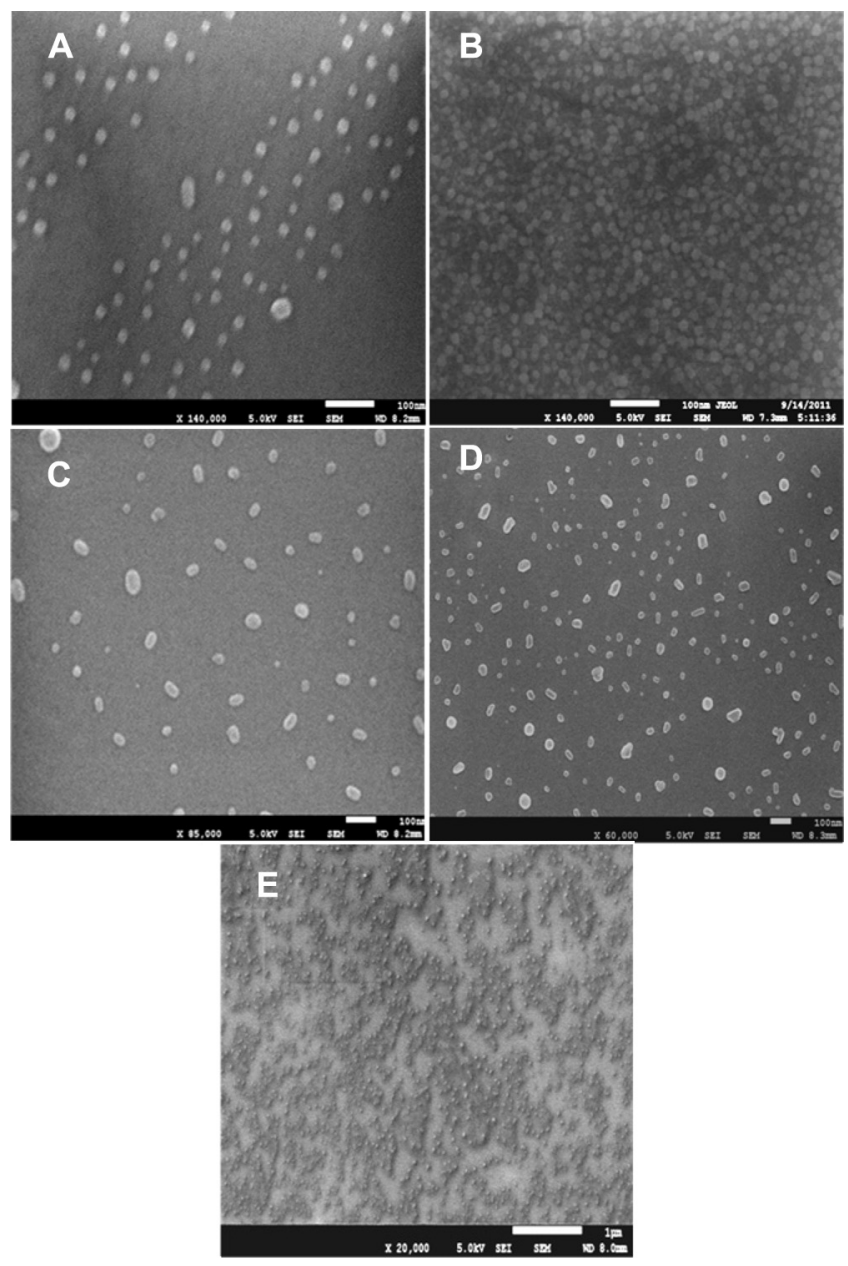

Figure SI Size analysis of permeation-enhanced drug-loaded nanoparticle. Representative field emission scanning electron microscopic image of permeation-enhanced drugloaded nanoparticles. (A) 4 wt\%, (B) 4.5 wt\%, (C) 5 wt\%, (D) 5.5 wt\%, and (E) 6 wt\% oleic acid (chemical permeation-enhanced) preparations.

Table SI Physicochemical characterization of permeation-enhanced dual drug-loaded nanoparticle preparations in different ratios of oleic acid concentration

\begin{tabular}{llll}
\hline Formulations & Size $(\mathbf{n m})$ & PDI & Zeta potential $(\mathbf{m V})$ \\
\hline Without OA & 477.4 & $0.509 \pm 0.031$ & $-31.4 \pm 2.7$ \\
6.0 wt\% OA & 228.6 & $0.339 \pm 0.023$ & $-55.4 \pm 3.9$ \\
5.5 wt\% OA & 120.8 & $0.305 \pm 0.016$ & $-53.3 \pm 3.5$ \\
5.0 wt\% OA & 80.4 & $0.220 \pm 0.021$ & $-51.9 \pm 2.3$ \\
4.5 wt\% OA & 41.3 & $0.118 \pm 0.013$ & $-47.3 \pm 2.4$ \\
4.0 wt\% OA & 38.5 & $0.112 \pm 0.023$ & $-46.1 \pm 1.9$ \\
\hline
\end{tabular}

Abbreviations: PDI, polydispersity index; OA, oleic acid. 

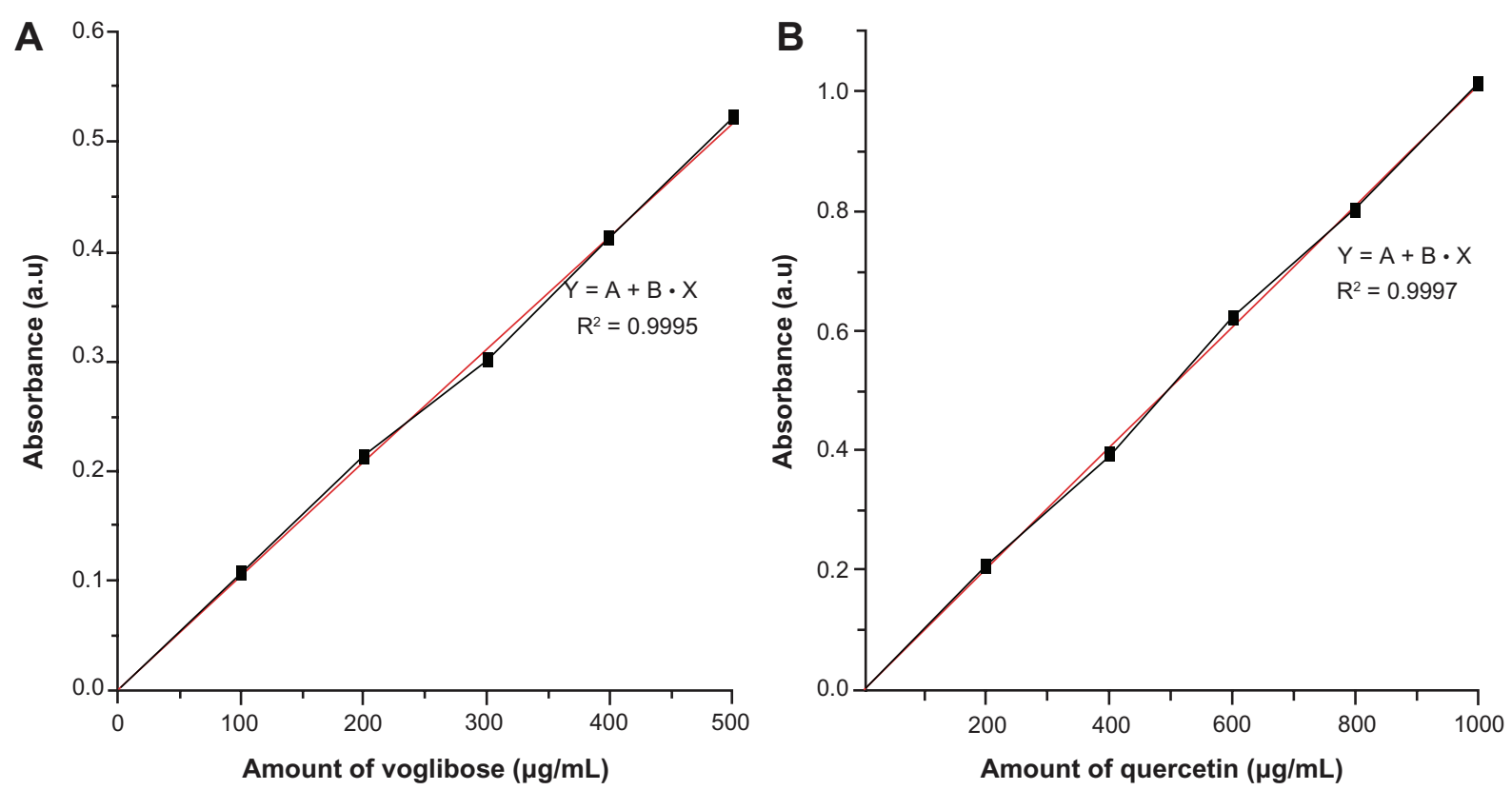

Figure S2 Calibration curve of drugs validated by UV-visible spectroscopic methods. This was found by plotting various concentrations of drugs versus absorbance with

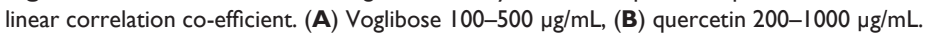

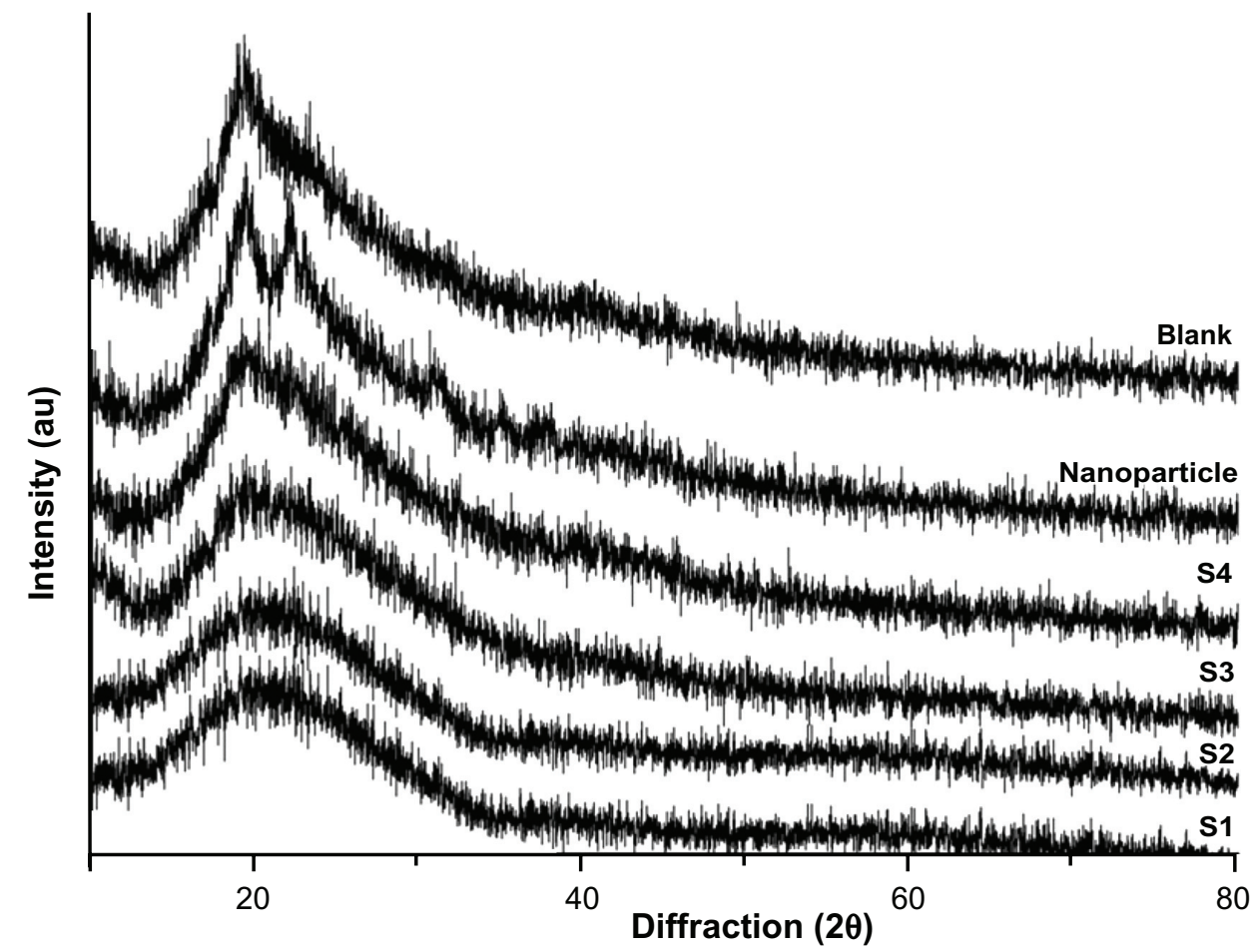

Figure S3 X-ray diffraction patterns of permeation-enhanced dual drug-loaded nanoparticle-incorporated cross-linked films (SI, S2, S3, and S4), noncross-linked film (blank), and drug-loaded nanoparticles. 


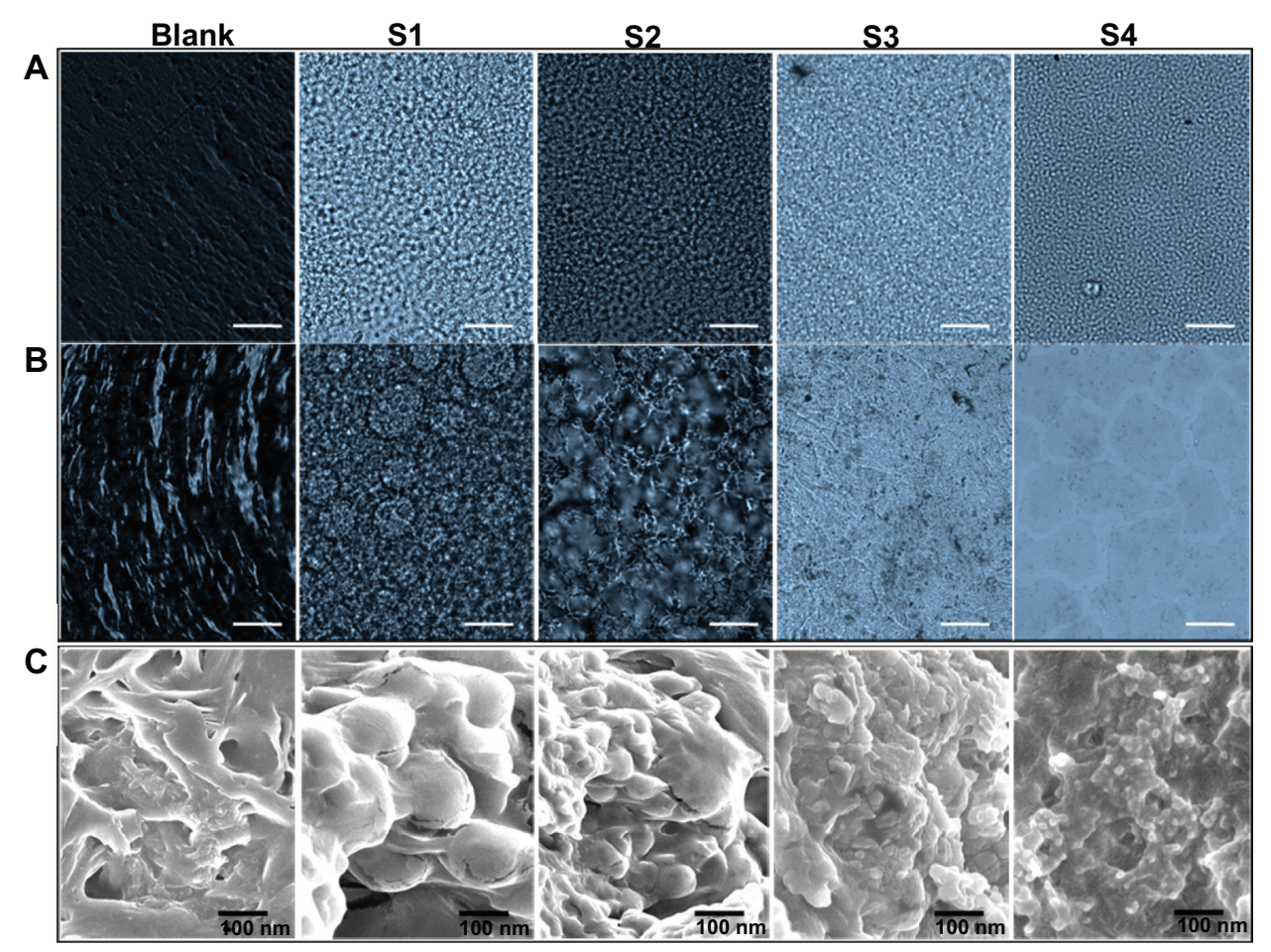

Figure S4 Optical microscopic images showing the morphology of blank film and nanoparticle-incorporated scaffold films (SI, S2, S3, and S4), before (A) and after (B) the release study. Scale bar, $20 \mu \mathrm{m}$ at 50× magnification. (C) Field emission scanning electron microscopic images of blank film and nanoparticle-incorporated scaffold films after release study. 
A

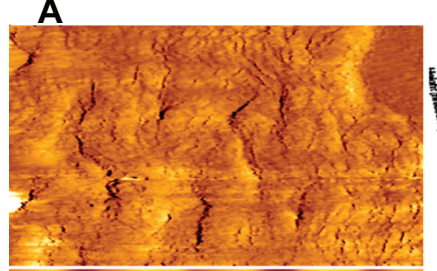

\section{B}
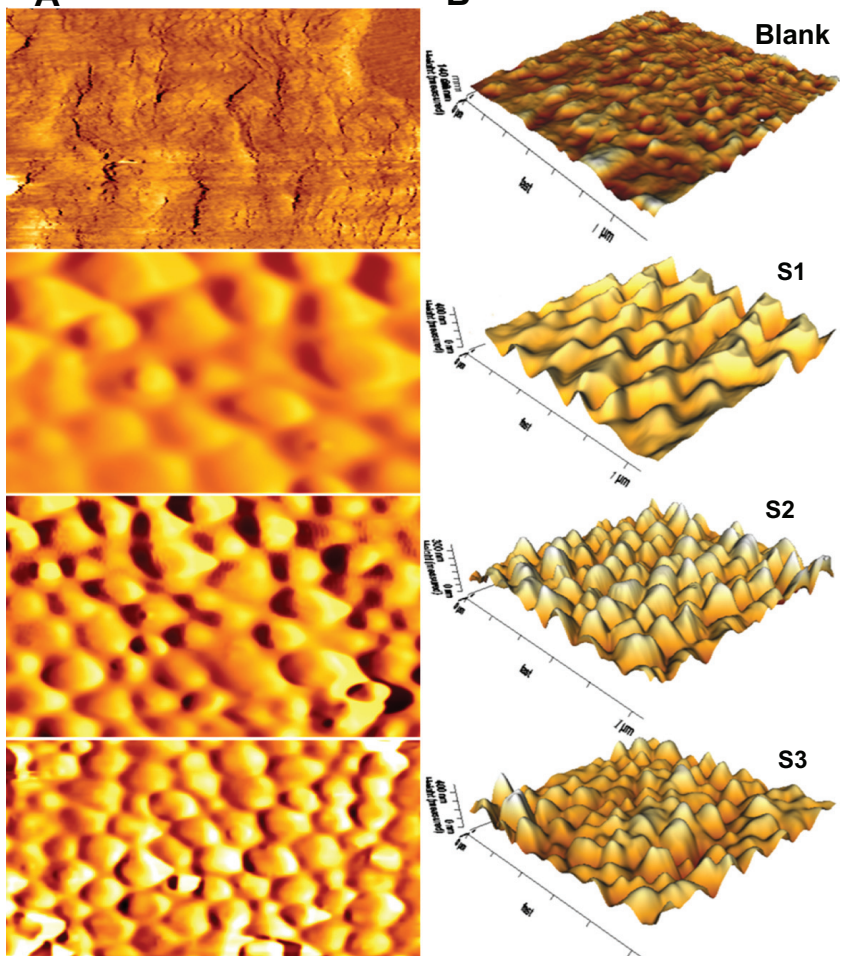

公

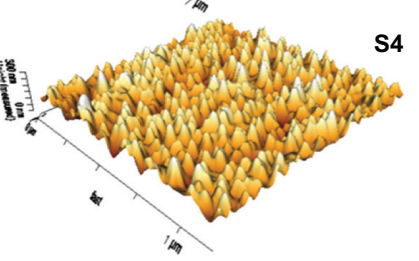

Figure S5 (A) Two-dimensional atomic force microscopic images showing the morphology of the swelling structure and surface properties of the blank, SI, S2, S3 and S4 films. (B) Corresponding three-dimensional atomic force microscopic images.

Notes: The blank film ensures the swelling structure has a relatively smooth surface and the nanoparticle-incorporated films have some projections, indicating the location of nanoparticles on the surface of the scaffold film. Scan size I $\times I \mu \mathrm{m}$.

International Journal of Nanomedicine

\section{Publish your work in this journal}

The International Journal of Nanomedicine is an international, peerreviewed journal focusing on the application of nanotechnology in diagnostics, therapeutics, and drug delivery systems throughou the biomedical field. This journal is indexed on PubMed Central, MedLine, CAS, SciSearch ${ }^{\circledR}$, Current Contents ${ }^{\circledR} /$ Clinical Medicine,
Journal Citation Reports/Science Edition, EMBase, Scopus and the Elsevier Bibliographic databases. The manuscript management system is completely online and includes a very quick and fair peer-review system, which is all easy to use. Visit http://www.dovepress.com/ testimonials.php to read real quotes from published authors. 